\title{
Greechie diagrams, nonexistence of measures in quantum logics, and Kochen-Specker-type constructions
}

\author{
K. Svozil \\ Institut für Theoretische Physik, University of Technology Vienna, Wiedner Hauptstrasse 8- \\ 10/136, A-1040 Vienna, Austria; e-mail: svozil@tph.tuwien.ac.at \\ J. Tkadlec \\ Department of Mathematics, Faculty of Electrical Engineering, Czech Technical University, \\ CZ-166 27 Prague, Czech Republic; e-mail: tkadlec@math.feld.cvut.cz
}

(Received 4 December 1995; accepted for publication 2 April 1996)

\begin{abstract}
We use Greechie diagrams to construct finite orthomodular lattices "realizable" in the orthomodular lattice of subspaces in a three-dimensional Hilbert space such that the set of two-valued states is not "large" (i.e., full, separating, unital, nonempty, resp.). We discuss the number of elements of such orthomodular lattices, of their sets of (ortho)generators and of their subsets that do not admit a "large" set of two-valued states. We show connections with other results of this type.
\end{abstract}

\section{INTRODUCTION}

Quantum logic, as it has been pioneered by Birkhoff and von Neumann [2], is usually derived from Hilbert space. There, the logical primitives, such as propositions and the logical operators "and", "or" and "not" are defined by Hilbert space entities. For instance, consider the threedimensional, real Hilbert space $\mathbf{R}^{3}$ with the usual scalar product $(v, w):=\sum_{i=1}^{3} v_{i} w_{i}, v, w \in \mathbf{R}^{3}$. There, any proposition is identified with a subspace of $\mathbf{R}^{3}$. For instance, the zero vector corresponds to a false statement. Any line spanned by a nonzero vector corresponds to the statement that the physical system is in the pure state associated with the vector. Any plane formed by the linear combination of two (noncolinear) vectors $v, w$ corresponds to the statement that the physical system is either in the pure state $v$ or in the pure state $w$. The whole Hilbert space $\mathbf{R}^{3}$ corresponds to the tautology (true propositions). The logical "and" operation is identified with the set theoretical intersection of two propositions; e.g., with the intersection of two lines. The logical "not" operation, or the "complement", is identified with taking the orthogonal subspace; e.g., the complement of a line is the plain orthogonal to that line.

In this top-down approach, one arrives at a propositional calculus that resembles the classical one, but differs from it in several important aspects. It has a non-Boolean, i.e., nondistributive, algebraic structure. Furthermore, as has first been pointed out by Kochen and Specker in the context of partial algebras $[25,10,11]$, there exist certain finite sets of lines, such that the associated propositional structure cannot be classically embedded. That is, there does not exist any classical, i.e., two-valued, measure which could be interpreted as the fact that propositions are either "true" (三 measure value 1) or "false" (三 measure value 0). The Kochen and Specker original construction used 117 lines. The number of lines has been subsequently reduced [16, 17, 13, 24]. These constructions are examples of propositional structures without any two-valued measures.

In this paper we shall deal with the following questions: which orthomodular structure - finite or infinite - underlies the Kochen-Specker construction. The question can be approached from two different viewpoints: (i) Which minimal set of propositions generates some Kochen-Specker-type configurations? By "generate" we mean the construction of the propositional structure containing it. (ii) What is the minimal propositional structure containing some sort of Kochen-Specker-type configuration? In particular, is it finite or infinite? 


\section{BASIC NOTIONS}

The following definition gives two main concepts of a propositional structure. tions.

Definition 2.1: An orthomodular poset is a structure $(P, \leq,, 0,1)$ fulfilling the following condi-

(1) $(P, \leq)$ is a partial ordered set such that $0 \leq a \leq 1$ for every $a \in P$.

(2) ' $: P \rightarrow P$ is an orthocomplementation, i.e., for every $a, b \in P$ : (a) $a^{\prime \prime}=a$, (b) $a \leq b$ implies $b^{\prime} \leq a^{\prime},(\mathrm{c}) a \vee a^{\prime}=1$.

(3) If $a \leq b^{\prime}$ then the supremum $a \vee b$ exists in $P$.

(4) If $a \leq b$ then there is an element $c \in L$ such that $c \leq a^{\prime}$ and $b=a \vee c$ (the orthomodular law).

An orthomodular lattice is an orthomodular poset that is a lattice.

Elements $a, b$ of an orthomodular poset are called orthogonal (denoted by $a \perp b$ ) if $a \leq b^{\prime}$. A subset $O$ of an orthomodular poset is called orthogonal if every pair of its elements is orthogonal.

Definition 2.2: Let $P_{1}, P_{2}$ be orthomodular posets. $P_{1}$ is orthorepresentable in $P_{2}$ if there is a mapping (called orthoembedding) $h: P_{1} \rightarrow P_{2}$ such that for every $a, b \in P_{1}$,

(1) $h(0)=0$,

(2) $h\left(a^{\prime}\right)=h(a)^{\prime}$,

(3) $a \leq b$ if and only if $h(a) \leq h(b)$, and

(4) $h(a \vee b)=h(a) \vee h(b)$ whenever $a \perp b$.

$P_{1}$ is representable in $P_{2}$ if there is a mapping (called embedding) $h: P_{1} \rightarrow P_{2}$ such that $h$ is orthoembedding and for every $a, b \in P_{1}$,

(4') $h(a \vee b)=h(a) \vee h(b)$.

The set $h\left(P_{1}\right)$ is then called an (ortho) representation of $P_{1}$ in $P_{2}$.

A suborthoposet (subortholattice, resp.) is a subset such that the identity mapping is orthoembedding (embedding, resp.).

Boolean subalgebra of an orthomodular poset is a suborthoposet that is a Boolean algebra. Block is a maximal Boolean subalgebra.

As we will see later, there are lattices $L_{1}, L_{2}$ such that $L_{1}$ is a suborthoposet but not a subortholattice of $L_{2}$. On the other hand, a suborthoposet of an orthomodular lattice need not be a lattice.

Definition 2.3: Let $L$ be an orthomodular lattice, $G, \bar{L} \subseteq L$ and let us denote by $L(G)[P(G)$, resp.] the least subortholattice (suborthoposet, resp.) of $L$ containing $G$. We say that $G$ generates (orthogenerates, resp.) $\bar{L}$ if $\bar{L} \subseteq L(G)(\bar{L} \subseteq P(G)$, resp.).

$P(G)$ and $L(G)$ can be explicitly defined by the following process: $P(G)=\bigcup_{n=0}^{\infty} P_{n}(G), L(G)=$ $\bigcup_{n=0}^{\infty} L_{n}(G)$, where $P_{0}(G)=L_{0}(G)=G$ and, for every natural number $n$ :

$$
\begin{aligned}
& L_{n+1}(G)=\left\{\bigvee O ; O \text { is a finite subset of } L_{n}(G) \cup L_{n}(G)^{\prime}\right\}, \\
& P_{n+1}(G)=\left\{\bigvee O ; O \text { is a finite orthogonal subset of } P_{n}(G) \cup P_{n}(G)^{\prime}\right\}
\end{aligned}
$$

( $M^{\prime}$ denotes the set $\left\{a^{\prime} ; a \in M\right\}$ ). Hence, every countable set $G$ generates a countable subortholattice and orthogenerates a countable suborthoposet.

A very useful tool for constructing and representing some orthomodular posets is the so-called Greechie diagram.

Definition 2.4: A diagram is a pair $(V, E)$, where $V \neq \emptyset$ is a set of vertices (usually drawn as points) and $E \subseteq \exp V \backslash\{\emptyset\}$ is a set of edges (usually drawn as line segments connecting corresponding points).

Let $n \geq 2$ be a natural number. A loop of order $n$ in a diagram $(V, E)$ is a sequence $\left(e_{1}, \ldots, e_{n}\right) \in$ $E^{n}$ of mutually different edges such that there are mutually different vertices $v_{1}, \ldots, v_{n}$ with $v_{i} \in e_{i} \cap e_{i+1}\left(i=1, \ldots, n, e_{n+1}=e_{1}\right)$.

A Greechie diagram is a diagram fulfilling the following conditions: 


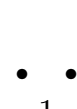

1

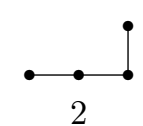

2

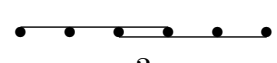

3

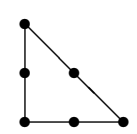

4

FIG. 1: Examples of diagrams that are not Greechie diagrams.

(1) Every vertex belongs to at least one edge.

(2) If there are at least two vertices then every edge is at least two-element.

(3) Every edge that intersects with another edge is at least three-element.

(4) Every pair of different edges intersects in at most one vertex.

(5) There is no loop of order 3.

Some examples of diagrams which are not Greechie diagrams are given in Fig. 1-these examples violates exactly one of conditions (2)-(5) in the above definition. (We usually do not denote oneelement edges.) The condition (4) states that in Greechie diagrams there is no loop of order 2.

Before we present the representation theorem let us recall that an atom in an orthomodular poset $P$ is a minimal element of $P \backslash\{0\}$.

Theorem 2.5: For every Greechie diagram with only finite edges there is exactly one (up to an isomorphism) orthomodular poset such that there are one-to-one correspondences between vertices and atoms and between edges and blocks that preserve incidence relations. A Greechie diagram does not contain any loop of order 4 if and only if the corresponding orthomodular poset is a lattice.

The proof can be found, e.g., in [14]. Let us reserve the notion Greechie logic for an orthomodular poset that can be represented by a Greechie diagram with only finite edges. It is easy to see that such an orthomodular poset does not contain any infinite chain, hence every its element is a supremum of a finite orthogonal set of atoms.

Let us remark that there are finite orthomodular posets not representable by Greechie diagramsintersections of blocks might be greater than a four-element Boolean subalgebra and hence the condition (4) of Definition 2.4 cannot be fulfilled. On the other hand, every orthomodular poset with only finite and at most three-atomic blocks (the case we are interested about) is a Greechie logic.

We will have a special interest about the following example.

Definition 2.6: The three-dimensional Hilbert logic $\mathrm{H}_{3}$ is the orthomodular lattice of linear subspaces of $\mathbf{R}^{3}$. The ordering is given by inclusion and the orthocomplementation is given by $a^{\prime}=\left\{v \in \mathbf{R}^{3} ; v \perp a\right\}$ for every $a \in H_{3}$.

The least element of $H_{3}$ is $0=\{(0,0,0)\}$, the greatest element of $H_{3}$ is $1=\mathbf{R}^{3}$. Moreover $a \wedge b=a \cap b$ and $a \vee b=\operatorname{Sp}(a \cup b)$ for every $a, b \in H_{3}$, where $\operatorname{Sp}(G)$ is the span of $G$ in $\mathbf{R}^{3}$. [We will usually omit unnecessary parentheses, e.g., $\operatorname{Sp}(1,0,0)$ denotes $\operatorname{Sp}(\{(1,0,0)\})$.]

Every element of $H_{3} \backslash\{0,1\}$ is either an atom or a coatom, every block in $H_{3}$ is finite and at most three-element, every suborthoposet $P$ of $H_{3}$ is a Greechie logic and is uniquely determined by the set $A_{1}(P)$ of its one-dimensional atoms (lines):

$$
P=\{0,1\} \cup A_{1}(P) \cup A_{1}(P)^{\prime} .
$$

(There might be also two-dimensional atoms in $P$, e.g., if $P$ is four-element.) Moreover, for every set $G$ of lines in $H_{3}$ the set of lines of the orthomodular lattice $L(G)$ [orthomodular poset $P(G)$, resp.] generated (orthogenerated, resp.) by $G$ can be expressed as follows: $A_{1}(P(G))=\bigcup_{n=0}^{\infty} P_{n}$, $A_{1}(L(G))=\bigcup_{n=0}^{\infty} L_{n}$, where $P_{0}=L_{0}=G$ and, for every natural number $n$,

$$
\begin{aligned}
& L_{n+1}=L_{n} \cup\left\{(a \vee b)^{\prime} ; a, b \in L_{n}\right\}, \\
& P_{n+1}=P_{n} \cup\left\{(a \vee b)^{\prime} ; a, b \in P_{n} \text { such that } a \perp b\right\} .
\end{aligned}
$$




\section{TWO-VALUED STATES AND GREECHIE DIAGRAMS}

Let us present the main definition.

Definition 3.1: Let $P$ be an orthomodular poset and let $G \subset P$. A state $s$ on $G$ is a mapping $s: P \rightarrow[0,1]$ such that:

(1) $s(0)=0$

(2) $s(a) \leq s(b)$ whenever $a, b \in G$ with $a \leq b$,

(3) $\sum_{a \in O} s(a) \leq 1$ for every orthogonal set $O \subset G$,

(4) $\sum_{a \in O} s(a)=1$ for every orthogonal set $O \subset G$ with $\bigvee O=1$

A two-valued state is a state with values in $\{0,1\}$.

If $G=P$ then conditions (1)-(2) follows from conditions (3)-(4) and from the orthomodular law and, moreover, $s\left(a^{\prime}\right)=1-s(a)$ for every $a \in P$.

The Kochen-Specker construction gives an example of a propositional structure without any two-valued state. We will use a more general attempt and will ask whether there is a propositional structure without "enough" two-valued states. Originally, "enough" meant "at least one". We will use also the following properties of state space, which are important in quantum logic theories.

Definition 3.2: Let $P$ be an orthomodular poset and let $G \subseteq P$. A set $S$ of states on $G$ is called unital if for every $a \in G \backslash\{0\}$ there is a state $s \in S$ such that $s(a)=1$;

separating if for every $a, b \in G$ with $a \neq b$ there is a state $s \in S$ such that $s(a) \neq s(b)$;

full if for every $a, b \in G$ with $a \not z b$ there is a state $s \in S$ such that $s(a)>s(b)$.

Existence of a unital set of states means that every proposition that is not a tautology is sometimes false. Existence of a separating set of states means that a different propositions are distinguishable. Existence of a full set of two-valued states means that if some proposition does not imply another, then there is such a state that the first is true while the second is not. These properties are largely studied. An orthomodular poset with a full set of two-valued states is called a concrete logic (see, e.g., [19]), an orthomodular poset with a separating set of two-valued states is called a partition logic - this notion is within orthomodular posets equivalent to the notion of automaton logic (see, e.g., [21, 22, 23]).

It is easy to see that a full set of states is separating and that a separating set of two-valued states is unital. Before we give examples demonstrating differences in the above-defined notions let us give some criteria, how we can verify whether an orthomodular poset given by a Greechie diagram has "enough" two-valued states.

Definition 3.3: Let $P$ be an orthomodular poset and let $A$ be the set of atoms in $P$. A weight $w$ on $A$ is a mapping $w: A \rightarrow[0,1]$ such that $\sum_{a \in O} w(a)=1$ for every maximal orthogonal set $O \subseteq P$. A two-valued weight is a weight with values in $\{0,1\}$.

Lemma 3.4: Let $P$ be a Greechie logic and let $A$ be the set of atoms in $P$. Then there is a one-to-one correspondence between two-valued states $s$ on $P$ and two-valued weights $w$ on $A$ given by $w=s \mid A$.

Proof: Obvious.

Due to this correspondence we may (and will) identify states and weights and study only the values of states on the set of atoms. Since every maximal orthogonal set of atoms corresponds uniquely to a block, we need only to check that the sum of values of a state on every edge in a Greechie diagram is equal to 1.

Proposition 3.5: Let $P$ be a Greechie logic and let $A$ be the set of atoms in $P$. Then $P$ has a full set of two-valued states (i.e., $P$ is a concrete logic) if and only if for every pair $a_{1}, a_{2} \in P$ of different nonorthogonal atoms there is a two-valued weight $w$ on $A$ such that $w\left(a_{1}\right)=w\left(a_{2}\right)=1$. 
Proof: $\Rightarrow$ : Let $a_{1}, a_{2} \in A$ such that $a_{1} \not \not a_{2}$. Then $a_{1} \not \leq a_{2}^{\prime}$ and there is a two-valued state $s$ on $P$ such that $1=s\left(a_{1}\right)>s\left(a_{2}^{\prime}\right)=0$. Hence, $s\left(a_{2}\right)=1$ and, according to Lemma 3.4 , it suffices to take $w=s \mid A$.

$\Leftarrow$ : Let $b_{1}, b_{2} \in P$ such that $b_{1} \not \leq b_{2}$, i.e., $b_{1} \not \subset b_{2}^{\prime}$. There are orthogonal sets $A_{1}, A_{2} \neq \emptyset$ of atoms in $P$ such that $b_{1}=\bigvee A_{1}, b_{2}^{\prime}=\bigvee A_{2}$. According to Lemma 3.4, it suffices to prove that there are atoms $a_{1} \in A_{1}, a_{2} \in A_{2}^{\prime}$ and a weight $w$ on $A$ such that $w\left(a_{1}\right)=w\left(a_{2}\right)=1$. Let us suppose first that $A_{1} \cap A_{2}=\emptyset$. Then there are atoms $a_{1} \in A_{1}$ and $a_{2} \in A_{2}$ such that $a_{1} \neq a_{2}$ and $a_{1} \not \perp a_{2}$ and, due to our assumption, a weight $w$ on $A$ such that $w\left(a_{1}\right)=w\left(a_{2}\right)=1$. Let us suppose now that $A_{1} \cap A_{2} \neq \emptyset$. Then there is an atom $a_{1} \leq b_{1}, b_{2}^{\prime}$ and either there is an atom $a_{2} \neq a_{1}$ such that $a_{1} \not \perp a_{2}$, or $a_{1} \perp a$ for every atom $a \neq a_{1}$. In both cases there is a two-valued weight $w$ on $A$ such that $w\left(a_{1}\right)=1$; in the first case due to our assumption and in the second case we can put $w(a)=1$ iff $a=a_{1}$.

The situation for a separating set of states is much more complicated and we will state a criterion in a special case (which is in our interest here).

Proposition 3.6: Let $P$ be a Greechie logic with at most three-atomic blocks and let $A$ be the set of atoms in $P$. Then the set of two-valued states on $P$ is separating (i.e., $P$ is a partition logic) if and only if the following conditions hold.

(1) For every atom $a \in P$ there is a two-valued weight $w$ on $A$ such that $w(a)=1$.

(2) For every pair $a_{1}, a_{2} \in P$ of different nonorthogonal atoms there are two-valued weights $w_{+}, w_{-}$on $A$ such that $w_{+}\left(a_{1}\right)=w_{+}\left(a_{2}\right)$ and $w_{-}\left(a_{1}\right) \neq w_{-}\left(a_{2}\right)$.

Proof: $\Rightarrow$ : Let $a \in A$. Then $a \neq 0$ and there is a two-valued state $s$ on $P$ such that $1=s(a)>$ $s(0)=0$. Let $a_{1}, a_{2} \in A$ such that $a_{1} \neq a_{2}$ and $a_{1} \not \perp a_{2}$. Then also $a_{1} \neq a_{2}^{\prime}$ and there are twovalued states $s_{-}, s_{+}$on $P$ such that and $1=s_{-}\left(a_{1}\right)>s_{-}\left(a_{2}\right)=0,1=s_{+}\left(a_{1}\right)>s_{+}\left(a_{2}^{\prime}\right)=0$, i.e., $s_{+}\left(a_{1}\right)=s_{+}\left(a_{2}\right)$. The rest follows from Lemma 3.4.

$\Leftarrow$ : Let $b_{1}, b_{2} \in P$ such that $b_{1} \neq b_{2}$. Since every element of $P \backslash\{0,1\}$ is either an atom or a coatom, there are atoms $a_{1}, a_{2} \in P$ such that $b_{1} \in\left\{0, a_{1}, a_{1}^{\prime}, 1\right\}$ and $b_{2} \in\left\{0, a_{2}, a_{2}^{\prime}, 1\right\}$. If $a_{1}=a_{2}$ then there are two-valued weights $w_{+}, w_{-}$on $A$ such that $w_{+}\left(a_{1}\right)=1$ and $w_{-}\left(a_{1}\right)=0$. If $a_{1} \neq a_{2}$ then there are two-valued weights $w_{+}, w_{-}$on $A$ such that $w_{+}\left(a_{1}\right)=w_{+}\left(a_{2}\right)$ and $w_{-}\left(a_{1}\right) \neq w_{-}\left(a_{2}\right)$. In both cases there are, according to Lemma 3.4, two-valued states $s_{+}, s_{-}$on $P$ such that either $s_{+}\left(b_{1}\right) \neq s_{+}\left(b_{2}\right)$ or $s_{-}\left(b_{1}\right) \neq s_{-}\left(b_{2}\right)$.

Let us present a lemma, which might simplify to verify criteria in Proposition 3.6.

Lemma 3.7: Let $P$ be a Greechie logic and let $A$ be the set of atoms in $P$. If $W$ is an at least three-element set of two-valued weights on $A$ such that $\left\{w^{-1}(1) ; w \in W\right\}$ is a partition of $A$, then

(1) for every atom $a \in A$ there is a weight $w \in W$ such that $w(a)=1$;

(2) for every pair $a_{1}, a_{2} \in A$ there is a weight $w \in W$ such that $w\left(a_{1}\right)=w\left(a_{2}\right)$.

Proof: Obvious.

Let us remark that in Greechie diagrams it suffices to use the above conditions for every connected subdiagram separately (weights behave independently on nonconnected subgraphs). In terms of orthomodular posets we can use the following important notion.

Definition 3.8: Let $\mathcal{P}$ be a set of orthomodular posets such that $P_{1} \cap P_{2}=\{0,1\}$ for every $P_{1}, P_{2} \in \mathcal{P}$ with $P_{1} \neq P_{2}$. The horizontal sum $\sum_{P \in \mathcal{P}} P$ is defined as $\left(\bigcup_{P \in \mathcal{P}} P, \bigcup_{P \in \mathcal{P}} \leq_{P}\right.$ , $\left.\bigcup_{P \in \mathcal{P}}{ }^{\prime P}, 0,1\right)$.

More generally, we speak about the horizontal sum of $P_{i}, i \in I$. It is an abbreviation for saying that we take disjoint representations $\bar{P}_{i}$ of $P_{i}$ (e.g., $\left.\{i\} \times P_{i}\right)$, identify all $\overline{0}_{i}(i \in I)$ and all $\overline{1}_{i}(i \in I)$ and take $\sum_{i \in I} \bar{P}_{i}$. It is easy to see that a horizontal sum of orthomodular posets (orthomodular lattices, resp.) is an orthomodular poset (orthomodular lattice, resp.) and that a set of states is 
nonempty (unital, separating, full, resp.) on a horizontal sum if and only if it is nonempty (unital, separating, full, resp.) on every horizontal summand.

In a Greechie diagram every connected subdiagram corresponds to a horizontal summand. (In particular, every finite two-atomic block is a horizontal summand.) On the other hand, the horizontal sum of Greechie logics is a Greechie logic with the Greechie diagram, which is a (disjoint) union of summands with only one exception - we loose isolated vertices (these correspond to the trivial orthomodular poset $\{0,1\})$.

The notion of a horizontal sum is a special kind of the notion of pasting. We are not interested here in a general setting (see, e.g., [14]), thus we describe only special cases how we can obtain a new Greechie logic using this process. Greechie diagram of the pasting of Greechie logics $P_{i}(i \in I)$ for atoms $a_{i} \in P_{i}(i \in I)$ we obtain as follows: we take the disjoint union of Greechie diagrams of $P_{i}(i \in I)$, identify vertices corresponding to $a_{i}(i \in I)$ and, if some $a_{i}(i \in I)$ belong to a two-atomic block, we delete necessary vertices corresponding to such $a_{i}^{\prime}$ such that the condition (3) of Definition 2.4 is fulfilled. Greechie diagram of the pasting of Greechie logics $P_{i}(i \in I)$ for blocks $B_{i} \subseteq P_{i}(i \in I)$ with the same number of atoms we obtain as follows: we take the disjoint union of Greechie diagrams of $P_{i}(i \in I)$ and identify edges corresponding to $B_{i}(i \in I)$ (i.e., we identify also atoms in these blocks). It is easy to see that such pastings of (lattice) Greechie logics are (lattice) Greechie logics.

The notion of a horizontal sum is related also to the following notion.

Definition 3.9: Let $P$ be an orthomodular poset. The distance $d$ on $P$ is a mapping $d: P \times P \rightarrow$ $\mathbf{N} \cup\{\infty\}$ defined by:

$$
\begin{aligned}
d(a, b)= & \inf \left\{n \in \mathbf{N} ; \text { there are blocks } B_{1}, \ldots, B_{n} \text { in } P\right. \text { such that } \\
& \left.B_{i} \cap B_{i+1} \neq\{0,1\} \text { for } i=0, \ldots, n, B_{0}=\{a\}, B_{n+1}=\{b\}\right\} .
\end{aligned}
$$

The distance function defines the largest decomposition of $P$ into horizontal summands - the least summands are maximal subsets of $P \backslash\{0,1\}$ of elements with finite distances joined with $\{0,1\}$.

The following result we will use in the sequel.

Proposition 3.10: Every Greechie logic without any loop has a full set of two-valued states.

Proof: The distance function on $P$ decompose $P$ into the horizontal sum $\sum_{i \in I} P_{i}$ such that the distance of every pair of elements in every summand is finite. It suffices to prove fullness for every summand. According to Proposition 3.5, it suffices, for every $i \in I$ and for every pair $a_{1}, a_{2}$ of different nonorthogonal atoms in $P_{i}$, to find a weight $w$ on the set $A$ of atoms in $P_{i}$ such that $w\left(a_{1}\right)=w\left(a_{2}\right)=1$. Let us put $A_{n}=\left\{a \in A ; d\left(a, a_{1}\right)=n\right\}$ for every natural number $n$ and let us define $w$ by induction:

I. $w\left(a_{1}\right)=1$.

II. Let us suppose that there is a natural number $n \geq 0$ such that $w$ is defined on $A_{0} \cup \cdots \cup A_{n}$. Every element of $A_{n+1}$ belongs to some block $B$ in $P_{i}$ such that $B \cap A_{n} \neq \emptyset$. For every such block $B$ we have $B \cap A_{n}=\left\{a_{B}\right\}$. If $w\left(a_{B}\right)=1$, we put $w \mid B \cap A \backslash A_{n}=0$. If $w\left(a_{B}\right)=0$, we can choose ( $B$ has at least three atoms) properly a $b_{B} \in B \cap A \backslash A_{n}$ and put $w\left(b_{B}\right)=1, w \mid B \cap A \backslash\left\{b_{B}\right\}=0$. Properly means that if $n=d\left(a_{2}, a_{1}\right)-2$ then $b_{B}$ is chosen such that it does not belong to the same block as $a_{2}$ and if $n=d\left(a_{2}, a_{1}\right)-1$ then $b_{B}=a_{2}$.

Let us present examples demonstrating differences in properties of state space.

Proposition 3.11: Let us consider the following conditions:

(1) The set of two-valued states is full.

(2) The set of two-valued states is separating but not full.

(3) The set of two-valued states is unital but not separating.

(4) The set of two-valued states is nonempty but not unital.

(5) The set of two-valued states is empty. 


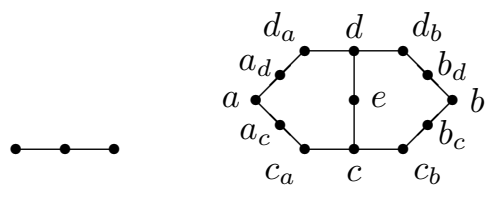

1
2

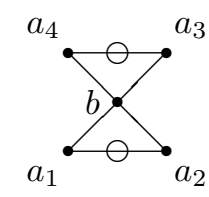

3

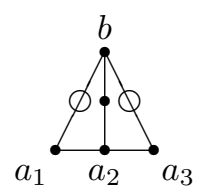

4

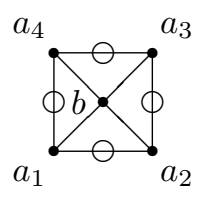

5

FIG. 2: Greechie diagrams of orthomodular posets with finite three-atomic blocks demonstrating differences of state spaces $(\mathrm{a} \bullet \bullet \bullet \mathrm{b}$ denotes diagram 2$)$.

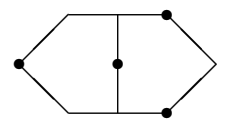

$s_{1}$

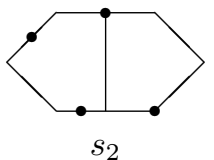

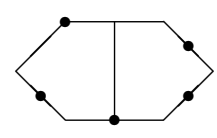

$s_{3}$

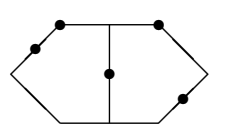

$s_{4}$

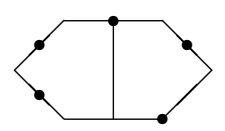

$s_{5}$

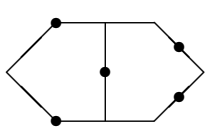

$s_{6}$

FIG. 3: Separating set of two-valued states on an orthomodular lattice from Fig. 2.2. (only atoms in which the corresponding state is equal to 1 are marked).

For each of the above conditions there is an orthomodular lattice with only finite three-atomic blocks, which fulfills it.

Proof: (1) See Fig. 2.1. It is a Boolean algebra, which obviously has a full set of two-valued states.

(2) See Fig. 2.2. For every two-valued state $s$ we have $s(a)+s(b) \leq\left(1-s\left(c_{a}\right)+1-s\left(d_{a}\right)+\right.$ $\left.1-s\left(c_{b}\right)+1-s\left(d_{b}\right)\right) / 2=(2-s(c)-s(d)) / 2 \leq 3 / 2$. Hence $s(a)+s(b) \leq 1$ and, according to Proposition 3.5, this orthomodular lattice has not a full set of two-valued states. The set $S_{1}=$ $\left\{s_{1}, s_{2}, s_{3}\right\}$ of states given in Fig. 3 fulfills conditions of Lemma 3.7. It can be checked that the set of all two-valued states "symmetric" to some state from $S$ distinguishes different nonorthogonal atoms. Hence, the set of two-valued states fulfills conditions of Proposition 3.6. A smaller example of a separating set of states is given in Fig. 3. We can express this orthomodular lattice as a partition logic on a six-element set of these states - see Fig. 4.1. (Compare with the representation on the 14-element set of states in [23].)

(3) See Fig. 2.3. Let us use the previous result. For every two-valued state $s$ with $s\left(a_{1}\right)=1$ we obtain $s\left(a_{2}\right)=s(b)=0$, hence $s\left(a_{4}\right)=1$. Using the symmetry we obtain $s\left(a_{1}\right)=s\left(a_{4}\right)$ for every two-valued state, hence the set of two-valued states is not separating. The unitality can be verified routinely.

(4) See Fig. 2.4. For every two-valued state $s$ there is an $i \in\{1,2,3\}$ such that $s\left(a_{i}\right)=1$ and therefore $s(b)=0$. Hence, the set of two-valued states is not unital. Existence of a two-valued state can be verified routinely. (Let us note that if we paste "sides of the triangle" not only for $b$ but for the whole block we obtain a smaller example with 25 atoms.)

(5) See Fig. 2.5. According to part (3) of this proof, $s\left(a_{1}\right)=s\left(a_{2}\right)=s\left(a_{3}\right)=s\left(a_{4}\right)$ for every two-valued state $s$. Hence all these values are equal to 0 and $s(b)=1$. The desired example we obtain by pasting this orthomodular lattice with the orthomodular lattice from Fig. 2.4 for $b$ 's or, more effectively, by pasting for blocks containing $b$ 's and $a_{2}$ 's.

\section{SUBORTHOLATTICES OF $\mathrm{H}_{3}$}

There are only several types of finite subortholattices of $H_{3}$. The following characterization of finite subortholattices of $H_{3}$ seems to be in a common knowledge (see, e.g., [8, Example 1.5.3]), but we do not know a proper reference for its proof.

Lemma 4.1: Let $L$ be a subortholattice of $H_{3}$ and let lines $a_{1}, a_{2}, a_{3}, b \in L$ be such that $a_{1}, a_{2}, a_{3}$ are mutually orthogonal and $b \not \subset a_{1}, a_{2}, a_{3}$. Then there is a line $c \in L$ such that $c \not \perp a_{3}$ and the angle $\angle\left(c, a_{3}\right)$ is greater than $\angle\left(b, a_{3}\right)$. 


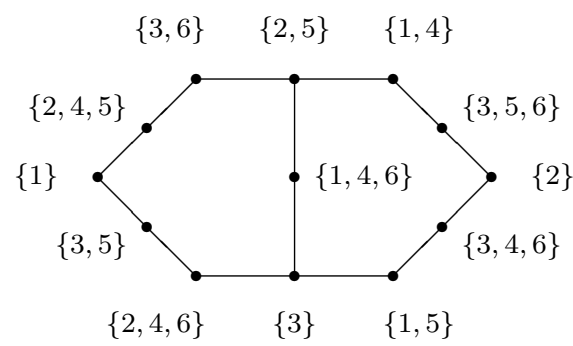

1

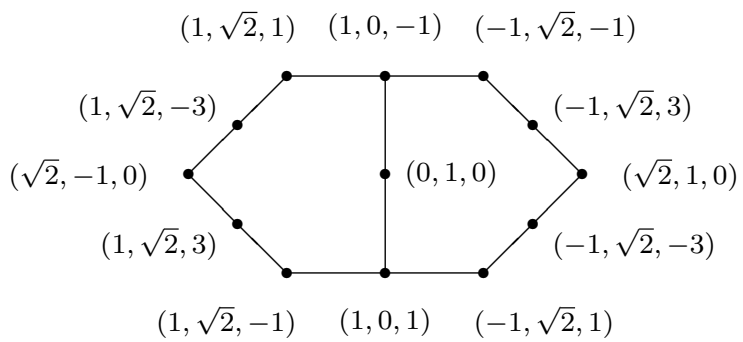

2

FIG. 4: Various representations of an orthomodular lattice from Fig. 2.2.

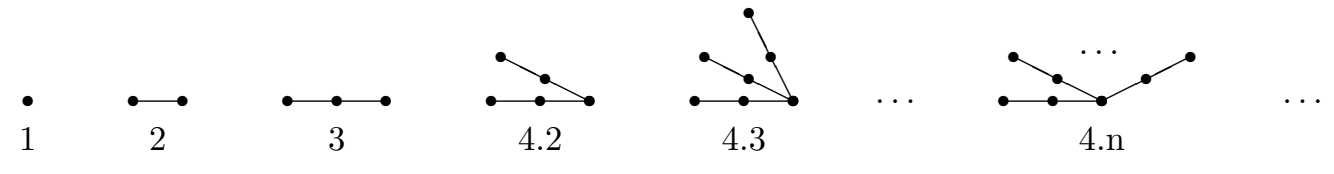

FIG. 5: Greechie diagrams of finite subortholattices of $H_{3}$.

Proof: Let us choose the system of coordinates such that $a_{1}=\operatorname{Sp}(1,0,0), a_{2}=\operatorname{Sp}(0,1,0)$, $a_{3}=\operatorname{Sp}(0,0,1), b=\operatorname{Sp}(x, y, z)$ such that $x, y, z>0$. Since $L$ is a subortholattice of $H_{3}$, the following elements belong to $L$ :

$$
\begin{aligned}
& \bar{b}=\left(a_{1} \vee a_{2}\right) \wedge b^{\prime}=\operatorname{Sp}(y,-x, 0) \\
& c=\left(a_{1} \vee a_{3}\right) \wedge(b \vee \bar{b})=\operatorname{Sp}\left(x+y^{2} / x, 0, z\right)
\end{aligned}
$$

Hence,

$$
0<\cos \angle\left(c, a_{3}\right)=\frac{z}{\sqrt{\left(x+y^{2} / x\right)^{2}+z^{2}}}<\frac{z}{\sqrt{x^{2}+y^{2}+z^{2}}}=\cos \angle\left(b, a_{3}\right) .
$$

Theorem 4.2: Let $L \subset H_{3}$ be a finite orthomodular lattice. Then $L$ is a subortholattice of $H_{3}$ if and only if exactly one of the following possibilities is fulfilled.

(1) $L=\{0,1\}$, i.e., $L$ is a one-atomic Boolean algebra.

(2) $L=\left\{0, a, a^{\prime}, 1\right\}$ for some line $a \in H_{3}$, i.e., $L$ is a two-atomic Boolean algebra.

(3) $L=\left\{0, a_{1}, a_{2}, a_{3}, a_{1}^{\prime}, a_{2}^{\prime}, a_{3}^{\prime}, 1\right\}$ for some orthogonal set $\left\{a_{1}, a_{2}, a_{3}\right\}$ of lines in $H_{3}$, i.e., $L$ is a three-atomic Boolean algebra.

(4) $L=\left\{0, a, a^{\prime}, 1\right\} \cup G \cup G^{\prime} \cup\{a \vee b ; b \in G\} \cup\left\{a^{\prime} \wedge b^{\prime} ; b \in G\right\}$ for some line $a \in H_{3}$ and some at least two-element set $G$ of mutually nonorthogonal atoms orthogonal to a, i.e., $L$ is a finite pasting of at least two three-atomic Boolean algebras for a given atom.

Proof: It is easy to see that each of these conditions excludes the others and gives a subortholattice of $H_{3}$. Let us suppose that there is a finite subortholattice $L$ of $H_{3}$ which fulfills no condition (1)-(4), and seek a contradiction. There are three mutually nonorthogonal lines $a, b, c \in L$. Let $d_{3}=(a \vee b)^{\prime} \in L$. Since $L$ is finite, there is a line $e \in L$ such that $L\left(e, d_{3}\right)$ is the greatest among all lines from $L$ nonorthogonal to $d_{3}$. Since $a \not \perp b$ there is a $d_{1} \in\{a, b\}$ such that $d_{1} \not \perp e, e^{\prime} \wedge d_{3}^{\prime}$. Let us put $d_{2}=d_{1}^{\prime} \wedge d_{2}^{\prime} \in L$. Hence, lines $d_{1}, d_{2}, d_{3}$ are mutually orthogonal and $e \not \perp d_{1}, d_{2}, d_{3}$. According to Lemma 4.1 , there is an element $f \in L$ such that $f \not \perp d_{3}$ and $\angle\left(f, d_{3}\right)<\angle\left(e, d_{3}\right)$ - this contradicts the selection of $e$.

Greechie diagrams of finite subortholattices of $H_{3}$ are given in Fig. 5 .

Corollary 4.3: Every finite subortholattice of $\mathrm{H}_{3}$ has a full set of two-valued states.

Proof: It follows from Theorem 4.2 and Proposition 3.10. 
As concerns infinite subortholattices of $\mathrm{H}_{3}$, there is a countable subortholattice of $\mathrm{H}_{3}$ without any two-valued states (e.g., generated by finite sets without any two-valued state - see Corollary 7.5). On the other hand, there are infinite subortholattices with a full set of two-valued states, e.g. infinite pastings of three-atomic Boolean algebras for a given atom [compare condition (4) of Theorem 4.2]. It seems to be an open problem whether there is an infinite subortholattice of $H_{3}$ which is not of this type and which has a two-valued state. Moreover, there might be an interesting connection between the nonexistence of a two-valued state and density in $\mathbf{R}^{3}$. This might give better insight into the nature of subortholattices of $H_{3}$ and the connection with famous Gleason theorem [5, 19], which (among other things) states that there is no two-valued state on $H_{3}$.

It should be noted that Greechie diagrams of subortholattices of $H_{3}$ are relatively "complex" the distance of every pair of elements is at most 2 (every pair of different lines has a common orthogonal line). Hence, it is usually difficult to give a Greechie diagram of an infinite subortholattice of $\mathrm{H}_{3}$.

\section{REALIZABILITY IN $\boldsymbol{H}_{3}$}

The study of finite suborthoposets of $H_{3}$ is more complicated. We would like to know whether a Greechie logic is orthorepresentable in $H_{3}$. The first problem erases with the intrinsic geometrical structure of $\mathrm{H}_{3}$.

Definition 5.1: Let $P$ be an orthomodular poset. We say that $P$ is weakly realizable in $H_{3}$ if there is a mapping $h: P \rightarrow H_{3}$ such that, for every $a, b \in P$,

(1) $h(0)=0$

(2) $h\left(a^{\prime}\right)=h(a)^{\prime}$

(3) $h(a) \leq h(b)$ whenever $a \leq b$; and

(4) $h(a) \neq 0$ whenever $a \neq 0$.

If, moreover, the mapping $h$ fulfills for every $a, b \in P$ the following condition

(4') $h(a) \neq h(b)$ whenever $a \neq b$,

we say that $P$ is realizable. The set $h(P)$ is called a (weak) realization of $P$ in $H_{3}$.

Weak realizability means that all orthogonality relations remains true in the images, and, since every nonzero element has a nonzero image, if the set of two-valued states on $G \subseteq P$ is empty (not unital, resp.) then the set of two-valued states on $h(G)$ is empty (not unital. resp.), too. Realizability means that, moreover, the mapping is one-to-one. Hence, if the set of two-valued states on $G \subseteq P$ is not separating (full, resp.), then the set of two-valued states on $h(G)$ is not separating (full, resp.), too. A realization need not be a suborthoposet because a new orthogonal pairs might appear in the images.

Let us give a characterization of orthomodular posets weakly realizable in $H_{3}$.

Lemma 5.2: Let $P_{\mathcal{P}}$ be the pasting of a set $\mathcal{P}$ of orthomodular posets and let there is a mapping $h: P_{\mathcal{P}} \rightarrow H_{3}$ such that $h(P)$ is a weak realization of $P$ for every $P \in \mathcal{P}$. Then $h\left(P_{\mathcal{P}}\right)$ is a weak realization of $P_{\mathcal{P}}$ in $H_{3}$. In particular, every horizontal sum of orthomodular posets weakly realizable in $\mathrm{H}_{3}$ is weakly realizable in $\mathrm{H}_{3}$.

Proof: Obvious.

Proposition 5.3: An orthomodular poset is weakly realizable in $\mathrm{H}_{3}$ if and only if every its block is finite and at most three-atomic.

Proof: $\Rightarrow$ : Every orthogonal set of nonzero elements in an orthomodular poset $P$ corresponds to an orthogonal set of nonzero elements in $H_{3}$. Since such a set in $H_{3}$ is at most three-element, every block of $P$ is finite with at most three atoms.

$\Leftarrow$ : Let $P$ be an orthomodular poset with only finite, at most three-atomic blocks. Let us decompose $P$ into the horizontal sum $\sum_{i \in I} P_{i}$ of minimal horizontal summands. Let us choose a 


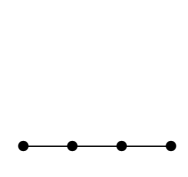

1

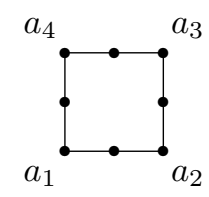

2

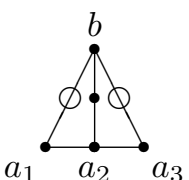

3

FIG. 6: Greechie diagrams of some orthomodular posets nonrealizable in $H_{3}(\mathrm{a} \bullet \bigcirc \bullet \mathrm{b}$ is an abbreviation of the Greechie diagram in Fig. 2.2.).

line $l \in H_{3}$ and let us define a mapping $h_{i}$ for every $i \in I$ as follows: $h_{i}(0)=0, h_{i}(1)=1$; if $P_{i}$ is four-element, then let us take an atom $a_{i} \in P_{i}$ and put $h_{i}\left(a_{i}\right)=l, h_{i}\left(a_{i}^{\prime}\right)=l^{\prime}$; if $P_{i}$ has more than four elements then every its block has three atoms and we put $h_{i}(a)=l, h_{i}\left(a^{\prime}\right)=l^{\prime}$ for every atom $a \in P_{i}$. It is easy to see that $h_{i}\left(P_{i}\right)$ is a weak realization of $P_{i}$ in $H_{3}$ and that $\bigcup_{i \in I} h_{i}\left(P_{i}\right)$ is a weak realization of $P$ in $H_{3}$.

The situation with realizability is more difficult and we do not know a characterization of it. Some results we will present in the next section. Let us present now another necessary condition.

Proposition 5.4: Every orthomodular poset realizable in $\mathrm{H}_{3}$ is a lattice.

Proof: Let us suppose that $P$ is an orthomodular poset with a loop of order 4 realizable in $H_{3}$ and seek a contradiction. There are nonzero mutually different elements $a_{1} \perp a_{2} \perp a_{3} \perp a_{4} \perp a_{1}$ in $P$ (see Fig. 6.2). Since for every pair of different nonzero elements there is only one nonzero element in $H_{3}$ orthogonal to them, $a_{1}=a_{3}$-a contradiction.

Examples of orthomodular posets nonrealizable in $H_{3}$ are given in Fig. 6 . The first has a fouratomic block, the second is not a lattice. The third example is much more subtle an depends on the following intrinsic property of $H_{3}$.

Lemma 5.5: Let $L$ be a realization of an orthomodular lattice given in Fig. 2.2. Then $\angle(a, b) \in$ $\langle\arccos 1 / 3, \pi / 2)$. On the other hand, for every $\alpha \in\langle\arccos 1 / 3, \pi / 2)$ there is a realization of $L$ such that $\angle(a, b)=\alpha$.

Proof: [See also [12].] Let us choose a coordinate system such that $c=\operatorname{Sp}(1,0,0), d=\operatorname{Sp}(0,1,0)$. Hence $e=\operatorname{Sp}(0,0,1)$. Since $c_{a} \perp c$ and $d_{b} \perp d$, there are $x, y \in \mathbf{R} \backslash\{0\}$ such that

$$
c_{a}=\operatorname{Sp}(0, y, 1), \quad d_{b}=\operatorname{Sp}(x, 0,1) .
$$

Since $c_{b} \perp c, c_{a}$ and $d_{a} \perp d, d_{b}, a \perp c_{a}, d_{a}$ and $b \perp c_{b}, d_{b}$, we obtain

$$
\begin{array}{cc}
c_{b}=\operatorname{Sp}(0,-1, y), & d_{a}=\operatorname{Sp}(-1,0, x), \\
a=\operatorname{Sp}(x y,-1, y), & b=\operatorname{Sp}(-1, x y, x) .
\end{array}
$$

Thus, using an elementary calculus,

$$
\cos \angle(a, b)=\frac{|x y|}{\sqrt{\left(1+x^{2}+x^{2} y^{2}\right)\left(1+y^{2}+x^{2} y^{2}\right)}} \in(0,1 / 3\rangle .
$$

For an arbitrary $\alpha \in\langle\arccos 1 / 3, \pi / 2)$ we can solve this equation and obtain, e.g.,

$$
x=y=\sqrt{\frac{1 / \cos \alpha-1}{2}-\sqrt{\left(\frac{1 / \cos \alpha}{2}\right)^{2}-1} .}
$$


For $\alpha=\arccos 1 / 3$ we have exactly one realization (two different solutions given by the symmetry of the Greechie diagram). In Fig. 4.2 there is an example such that symmetries of the realization are easily seen (with respect to the axis $o$ of $a$ and $b$ and to planes $\operatorname{Sp}\{a, b\}, \operatorname{Sp}\{o, a \times b\}$ ). For $\alpha \in(\arccos 1 / 3, \pi / 2)$ we have two different realizations (each symmetric with respect to the axis of $a$ and $b)$.

The orthomodular lattice given in Fig. 6.3 is not realizable, because for every triple $a_{1}, a_{2}, a_{3} \in$ $H_{3}$ of mutually orthogonal nonzero elements and for every $b \in H_{3}$ there is an $i \in\{1,2,3\}$ such that $\angle\left(b, a_{i}\right) \leq \arccos 1 / \sqrt{3}$.

Let us note that in [12] the above lemma is stated also for $\alpha=\pi / 2$. This is not true, because then either $x=0$ or $y=0$ and we obtain only a weak realization.

\section{SUBORTHOPOSETS OF $\boldsymbol{H}_{3}$}

We would like to present examples of orthomodular lattices orthorepresentable in $H_{3}$. To ensure that an orthomodular lattice is orthorepresentable in $H_{3}$ it suffices to find its realization in $H_{3}$ such that there are not ordered (orthogonal, resp.) pairs other than it was intended. E.g., it can be easily verified that an orthomodular lattice given in Fig. 2.2 is orthorepresentable in $H_{3}$ (see Fig. 4.2). We present partial results which orthomodular lattices are orthorepresentable (realizable, resp.) in $H_{3}$. The idea of their proofs is that we can find uncountable many (continuum) weak realizations while only for a countable many of them some images coincide or, in case of orthorepresentability, give a new ordered (orthogonal, resp.) pair.

We show that there is a large class of infinite suborthoposets of $H_{3}$ with a full set of two-valued states.

Proposition 6.1: Every horizontal sum of countable many countable orthomodular lattices orthorepresentable (realizable, resp.) in $\mathrm{H}_{3}$ is orthorepresentable (realizable, resp.) in $\mathrm{H}_{3}$.

Proof: It suffices to prove this proposition for two summands (we can proceed by induction). Let $L_{1}, L_{2}$ be their orthorepresentations (realizations, resp.) in $H_{3}$. It suffices to prove that we can rotate $L_{2}$ to $\bar{L}_{2}$ such that $a_{1} \nsubseteq a_{2}$ and $a_{2} \nsubseteq a_{1}$ for every $a_{1} \in L_{1} \backslash\{0,1\}$ and for every $a_{2} \in \bar{L}_{2} \backslash\{0,1\}$, i.e. such that $l \nsubseteq \subseteq \bigcup\left(L_{1} \backslash\{1\}\right)$ for every line $l \in \bar{L}_{2}$. If $L_{2}=\{0,1\}$ then the proof is complete. Let us suppose that $L_{2} \neq\{0,1\}$. Then there is a line $l_{0} \in L_{2}$. Since $\bigcup\left(L_{1} \backslash\{1\}\right) \neq \mathbf{R}^{3}$ there is a line $\bar{l}_{0} \nsubseteq \subseteq\left(L_{1} \backslash\{1\}\right)$ and we can rotate $L_{2}$ such that $l_{0}$ goes to $\bar{l}_{0}$. Rotating now the image of $L_{2}$ around $\bar{l}_{0}$ we obtain an uncountable many possibilities while for only a countable many of them there is a line $\bar{l} \in \bar{L}_{2}$ such that $\bar{l} \subseteq \bigcup\left(L_{1} \backslash\{1\}\right)$. Indeed, for every $l \in L_{2}$ all possible positions of $\bar{l}$ in a unit sphere $S(0,1)$ in $\mathbf{R}^{3}$ form a circle $C$ with the center on $\bar{l}_{0}$, while, for every $a \in L_{1} \backslash\{1\}, a \cap S(0,1)$ is either a two-element set ( $a$ is a line) or a circle not identical to $C$; hence $a \cap S(0,1) \cap C$ is at most two-element.

Proposition 6.2: Every pasting for an atom of a pair of countable orthomodular lattices orthorepresentable (realizable, resp.) in $\mathrm{H}_{3}$ is orthorepresentable (realizable, resp.) in $\mathrm{H}_{3}$.

Proof: If we paste for an atom in a two-atomic block then we obtain a horizontal sum and the proof follows from Proposition 6.1. Let us suppose that we paste for atoms in three-atomic blocks. Let $L_{1}, L_{2}$ be orthorepresentations (realizations, resp.) in $H_{3}$ of given orthomodular lattices such that $L_{1} \cap L_{2} \ni l_{0}$ where $l_{0}$ represents the atom in both $L_{1}, L_{2}$ for which we paste. It suffices to prove that there is a rotation $\bar{L}_{2}$ of $L_{2}$ around the line $l_{0}$ such that $a_{1} \nsubseteq a_{2}$ and $a_{2} \nsubseteq a_{1}$ for every $a_{1} \in L_{1} \backslash\left\{0,1, l_{0}, l_{0}^{\prime}\right\}$ and for every $a_{2} \in L_{2} \backslash\left\{0,1, l_{0}, l_{0}^{\prime}\right\}$, i.e., such that $l \nsubseteq \bigcup\left(L_{1} \backslash\left\{1, l_{0}^{\prime}\right\}\right)$ for every line $l \in \bar{L}_{2}$. This gives only countable many restrictions to uncountable possible positions of $\bar{L}_{2}$, hence the proof is complete.

Corollary 6.3: Every countable Greechie logic with at most three-atomic blocks and without any loop is orthorepresentable in $\mathrm{H}_{3}$. 


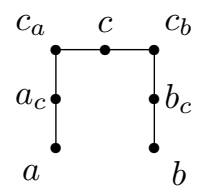

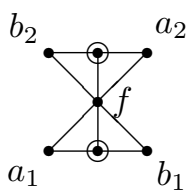

2

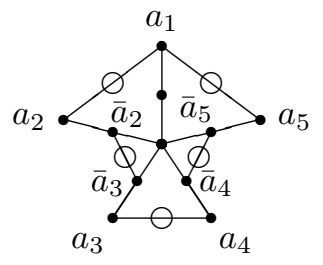

3

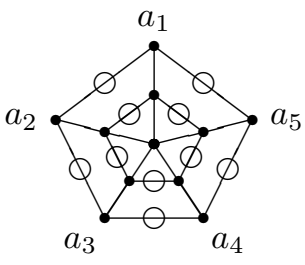

4

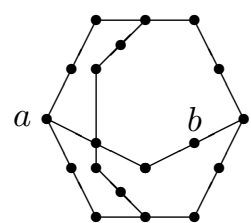

5

FIG. 7: Greechie diagrams of orthomodular lattices weakly realizable in $H_{3}$.

Proof: Every countable Greechie logic with only finite at most three-atomic blocks is a horizontal sum of subsequent countable pastings of finite three-atomic Boolean algebras for an atom. The rest follows from Theorem 4.2, Proposition 6.2 (using the induction) and Proposition 6.1.

According to Proposition 3.10, Greechie logics from the above Corollary have a full set of two-valued states.

Lemma 6.4: Let $L_{1}$ be a countable orthomodular lattice orthorepresentable (realizable, resp.) in $H_{3}$ and $L_{2}$ be an orthomodular lattice given in Fig. 7.1 such that $L_{1} \cap L_{2}=\left\{0, a, b, a^{\prime}, b^{\prime}, 1\right\}$ and $a \neq b$ are nonorthogonal atoms in $L_{1}$ (in its realization, resp.). Then the pasting of $L_{1}$ and $L_{2}$ is orthorepresentable (realizable, resp.) in $\mathrm{H}_{3}$.

Proof: Let us suppose that $L_{1}$ is an orthorepresentation (realization, resp.) in $H_{3}$ of a given orthomodular lattice. If $a$ ( $b$, resp.) is a two-dimensional subspace of $H_{3}$ then $a$ ( $b$, resp.) is a part of a four-element horizontal summand and this summand might be considered as a part of $L_{2}$. The proof then follows from Proposition 6.2. Let us suppose that $a, b$ are lines. Let us consider all atoms $c_{a} \leq a^{\prime}$. We have uncountable many possibilities that fill in the unit sphere $S(0,1)$ a circle $C_{a}$. Of course, $c_{a} \leq a^{\prime}$ and $a_{c}=a^{\prime} \wedge c_{a}^{\prime} \leq a^{\prime}$ but all other ordering of $c_{a}$ and $a_{c}$ with elements of $L_{1} \backslash\{0,1\}$ can be excluded if we exclude a countable many possibilities. Similarly, if positions of $c_{a}$ fill a circle $C_{a}$ then positions of $c_{b} \perp c_{a}, b$ fill a circle $C_{b} \subset b^{\prime}(a \not \perp b)$. Again, there is only a countable many positions of $c_{a}$ for which either $c_{b}$ or $b_{c}=b^{\prime} \wedge c_{b}^{\prime}$ is ordered with some element of $L_{1} \backslash\left\{0,1, b^{\prime}\right\}$. Finally, it can be shown that positions of $c$ fill a smooth curve on $S(0,1)$, which is not a circle. Hence, there is a possibility to choose $c_{a}$ such that we obtain the desired orthorepresentation (realization, resp.).

Proposition 6.5: Let $n \geq 5$ be a natural number and let $B_{1}, \ldots, B_{n}$ be finite 3-atomic Boolean algebras such that $B_{i} \cap B_{i+1}=\left\{0, a_{i}, a_{i}^{\prime}, 1\right\}$ for every $i \in\{1, \ldots, n\}$, where $B_{n+1}=B_{1}$ and $a_{1}, \ldots, a_{n}$ are mutually different atoms. Then the pasting of $\left\{B_{1}, \ldots, B_{n}\right\}$ (so-called $n$-cycle) is orthorepresentable in $\mathrm{H}_{3}$.

Proof: It follows from Proposition 6.2 and from Lemma 6.4.

\section{KOCHEN-SPECKER-TYPE CONFIGURATIONS}

We will give several examples of Kochen-Specker-type configurations which arise from Greechie diagrams. Some of these examples has been already used in the literature in the attempt to find a subset of $H_{3}$ without a two-valued state. We present the connection to Greechie diagrams (this gives a better geometric insight), show a nonexistence of a "large" set of two-valued states for various concepts, and, moreover, we do not stop in proving weak realizability but we discuss the real number of elements.

Proposition 7.1: There is a finite suborthoposet of $H_{3}$ such that the set of two-valued states on it is not full. 
Proof: Let us consider a suborthoposet $\mathrm{L}_{\text {of }} \mathrm{H}_{3}$ given in Fig. 4.2. It is an orthorepresentation of an orthomodular lattice given in Fig. 2.2, it is 28-element (13-atomic) and the set of two-valued states on $L$ is not full [see the proof of Proposition 3.11.(1)]. In fact, in the proof of Proposition 3.11.(1) it was shown that there is no two-valued state on the eight-element set $\left\{a, c_{a}, d_{a}, c, d, c_{b}, d_{b}, b\right\}$ such that $s(a)=s(b)=1$ (a reformulation of fullness-see Proposition 3.5). This orthomodular lattice can be orthogenerated, e.g., by the six-element set $\left\{a, c_{a}, c_{b}, b, d_{b}, d_{a}\right\}$ and generated, e.g., by the three-element set $\left\{a, c_{b}, d_{b}\right\}$.

Proposition 7.2: There is a finite suborthoposet of $\mathrm{H}_{3}$ such that the set of two-valued states on it is not separating.

Proof: Let us consider an orthomodular lattice given in Fig. 2.3. It is an orthomodular lattice without a separating set of two-valued states [see the proof of Proposition 3.11.(2)]. It has 56 elements (27 atoms) and a 17-element subset without a separating set of states (five marked and six "hidden" in every circle). It can be checked that it has the following realization (which forms a suborthoposet of $H_{3}$ given in Fig. 7.2 - points in circles denotes the middle elements of the diagram from Fig. 2.2): $f=\operatorname{Sp}(0,0,1), a_{1} \bullet \bullet \bullet b_{1}$ given by Fig. $4.2 ; a_{2} \bullet \bullet \bullet b_{2}$ we obtain from the representation on Fig. 4.2 rotating by $\pi / 2$ around $f$. There is a ten-element set of orthogenerators (e.g., $\left.\left\{a_{1}, b_{1}, c_{a 1}, c_{b 1}, d_{a 1}, d_{b 1}, f, c_{2}, c_{b 2}, d_{b 2}\right\}\right)$ and a four-element set of generators (e.g., $\left.\left\{a_{1}, c_{b 1}, d_{b 1}, c_{b 2}\right\}\right)$.

Let us note that we can take a realization of an orthomodular lattice given in Fig. 2.2, such that we obtain an orthorepresentation of the orthomodular lattice given in Fig. 2.3, but the set of (ortho)generators is larger in this case.

Proposition 7.3: There is a finite suborthoposet of $\mathrm{H}_{3}$ such that the set of two-valued states on it is not unital.

Proof: Let us consider an orthomodular lattice $L$ given in Fig. 7.3. It is an orthomodular lattice without a unital set of two-valued states. Indeed, for every two-valued state $s$ on $L$ with $s\left(a_{1}\right)=1$ we have $s(f)=s\left(a_{2}\right)=s\left(a_{5}\right)=0, s\left(\bar{a}_{2}\right)=s\left(\bar{a}_{5}\right)=1, s\left(\bar{a}_{3}\right)=s\left(\bar{a}_{4}\right)=0, s\left(a_{3}\right)=s\left(a_{4}\right)=1$ contradiction. It has 132 elements (65 atoms) and a 40-element subset without a unital set of states (six hidden in every circle and all marked $a_{i}$ 's and $\bar{a}_{i}$ 's). Let us find a weak realization of $L$. It can be done as follows: Put $f=\operatorname{Sp}(0,0,1), a_{1}=\operatorname{Sp}(1,0,0), \bar{a}_{1}=\operatorname{Sp}(0,1,0)$ and let $a_{k}, \bar{a}_{k}$ $(k=2, \ldots, 5)$ be images of $a_{1}, \bar{a}_{1}$ in rotations around $f$ about $k \cdot 72^{\circ}$. Find a realization of the orthomodular lattice given in Fig. 2.2 such that the angle of images of $a, b$ is $72^{\circ}$ (see the proof of Lemma 5.5) and rotate this realization to the following pairs of lines: $\left(a_{1}, a_{2}\right),\left(\bar{a}_{2}, \bar{a}_{3}\right),\left(a_{3}, a_{4}\right)$, $\left(\bar{a}_{4}, \bar{a}_{5}\right),\left(a_{5}, a_{1}\right)$ (i.e., $a$ goes to the first and $b$ to the second line for every pair). It can be checked that an orthomodular poset orthogenerated by this weak realization is finite. (In fact, it is a weak realization of an orthomodular lattice given in Fig. 7.4 by the same way.)

It can be shown that if we take the realization of the orthomodular lattice given in Fig. 2.2 such that the angle between $a$ and $b$ is equal to $72^{\circ}$ by the expression given in the proof of Lemma 5.5 as the first copy and if the second and the third copy arise by rotations around the axis of the plane given by $a$ and $b$ such that $b$ coincides with $a$ of the next copy, then some elements coincide:

$$
\begin{aligned}
\left(c_{a}, c, c_{b}, b_{c}, b, b_{d}, d_{b}\right)_{1} & =\left(d, d_{b}, d_{a}, a_{d}, a, a_{c}, c_{a}\right)_{2} \\
\left(c, d_{b}, d, d_{a}, e\right)_{1} & =\left(c_{a}, d, c, e, c_{b}\right)_{3} .
\end{aligned}
$$

(The index denotes the number of the copy.) Hence, the weak realization of the orthomodular lattice from the above proof gives a 29-element subset of $H_{3}$ without a unital set of two-valued states and the suborthoposet orthogenerated by it has 104 elements (51 atoms), is orthogenerated by a 16 -element set and generated by a four-element set (e.g., elements $a, c_{b}, d_{b}$ of some a $\bullet \bullet \bullet \mathrm{b}$ and some element from the inner "pentagon"). The "almost" Greechie diagram (20 points that belong to exactly one edge are for simplicity omitted) of this suborthoposet of $H_{3}$ (realization of the orthomodular lattice given in Fig. 7.4) is given in Fig. 8, with 


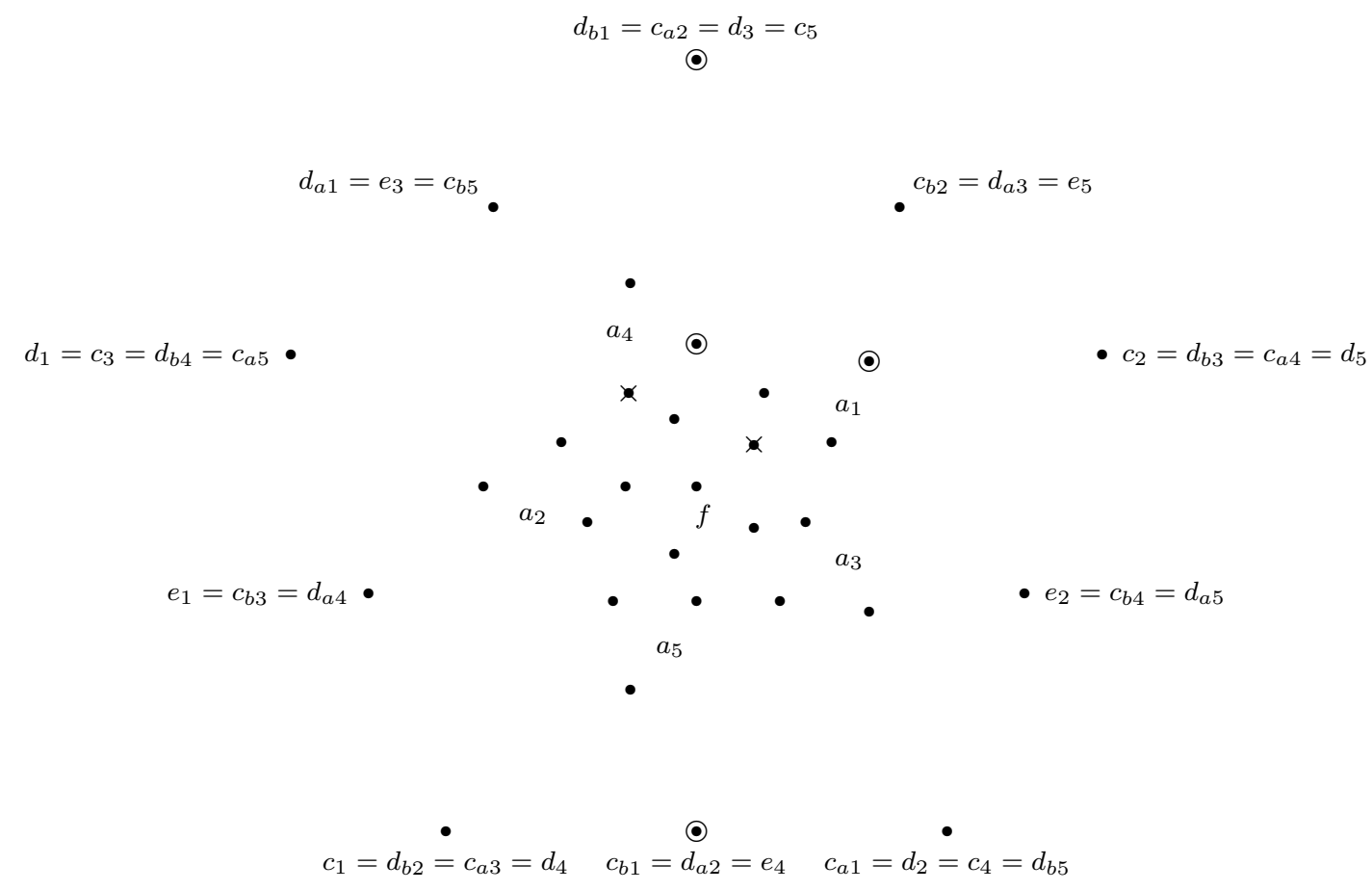

FIG. 8: "Almost" Greechie diagram of a suborthoposet of $H_{3}$ without a unital set of two-valued states.

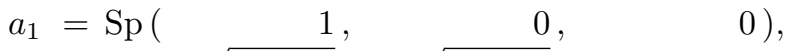

$$
\begin{aligned}
& a_{2}=\operatorname{Sp}(\sqrt{3-\sqrt{5}}, \quad \sqrt{5+\sqrt{5}}, \quad 0), \\
& a_{3}=\operatorname{Sp}(-\sqrt{3+\sqrt{5}}, \quad \sqrt{5-\sqrt{5}}, \quad 0), \\
& a_{4}=\operatorname{Sp}(-\sqrt{3+\sqrt{5}},-\sqrt{5-\sqrt{5}}, \quad 0), \\
& a_{5}=\operatorname{Sp}(\sqrt{3-\sqrt{5}},-\sqrt{5+\sqrt{5}}, \quad 0), \\
& c_{a 1}=\operatorname{Sp}(\quad 0,-\sqrt{-1+\sqrt{5}}, \quad 1), \\
& d_{a 1}=\operatorname{Sp}(\quad 0, \quad \sqrt{2}, \sqrt{-2+\sqrt{5}}), \\
& c_{1}=\operatorname{Sp}(\quad \sqrt{\sqrt{5}}, \quad \sqrt{2+\sqrt{5}}, \quad \sqrt{3+\sqrt{5}}), \\
& d_{1}=\operatorname{Sp}(\quad-\sqrt{\sqrt{5}},-\sqrt{-2+\sqrt{5}}, \quad \sqrt{2}), \\
& c_{b 1}=\operatorname{Sp}(-\sqrt{5+\sqrt{5}}, \quad \sqrt{3-\sqrt{5}}, 2 \sqrt{-2+\sqrt{5}}), \\
& d_{b 1}=\operatorname{Sp}(\quad \sqrt{\sqrt{5}},-\sqrt{-2+\sqrt{5}}, \quad \sqrt{2}), \\
& e_{1}=\operatorname{Sp}(\quad \sqrt{\sqrt{5}},-\sqrt{2+\sqrt{5}}, \quad \sqrt{3-\sqrt{5}}), \\
& c_{2}=\operatorname{Sp}(\quad-\sqrt{\sqrt{5}}, \quad \sqrt{2+\sqrt{5}}, \quad \sqrt{3+\sqrt{5}}), \\
& c_{b 2}=\operatorname{Sp}(\quad-\sqrt{\sqrt{5}},-\sqrt{2+\sqrt{5}}, \quad \sqrt{3-\sqrt{5}}), \\
& e_{2}=\operatorname{Sp}(\sqrt{5+\sqrt{5}}, \quad \sqrt{3-\sqrt{5}}, 2 \sqrt{-2+\sqrt{5}}), \\
& f=\operatorname{Sp}(\quad 0, \quad 0, \quad 1) \text {. }
\end{aligned}
$$

Elements of the 29-element subset without a unital set of two-valued states are all marked points that are not crossed, a set of orthogenerators is, e.g., the set of vertices of both pentagons with $a_{i}$ 's and with the middle point, a set of generators is marked by circles.

It should be noted that in [24, 27] there is an example of an 11-element set of lines orthogenerating a 25-element set of lines and a 76-element (37-atomic) suborthoposet of $H_{3}$ without a unital set of two-valued states. This suborthoposet is generated by a three-element set. The Greechie diagram of this example does not seem to provide an easy survey, hence we omit it. A more detailed description of this example is given in Sec. VIII. 


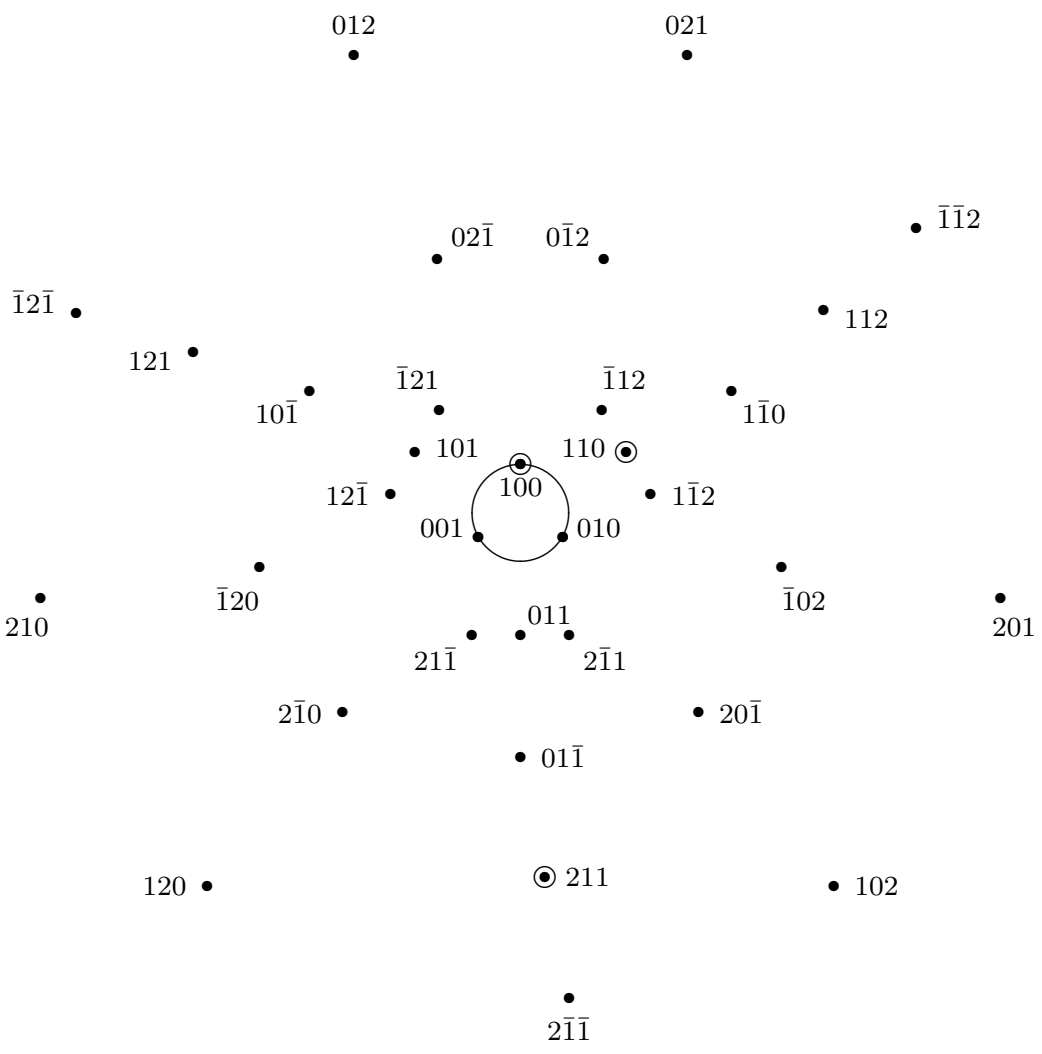

FIG. 9: "Almost" Greechie diagram of a suborthoposet of $H_{3}$ without any two-valued state [e.g., $1 \overline{1} 2$ denotes $\operatorname{Sp}(1,-1, \sqrt{2})]$.

Proposition 7.4: There is a finite suborthoposet of $H_{3}$ such that the set of two-valued states on it is empty.

Proof: Let us consider an orthomodular lattice $L$, which is the pasting of the orthomodular lattice given in Fig. 7.3 for $a_{1}$ and of the orthomodular lattice given in Fig. 7.4 for its middle point. It is an orthomodular lattice without any two-valued state. Indeed, if $s$ is a two-valued state on $L$ then $s\left(a_{1}\right)=0$ (see above). Analogously from the other diagram, $s\left(a_{1}\right)=1-$ a contradiction. It has 374 elements (186 atoms) and a 110-element subset without any two-valued state (six "hidden" in every circle and all marked except two of them $-a_{1}$ and $\left.\bar{a}_{1}\right)$. According to Proposition 6.2 , this orthomodular poset is weakly realizable in $H_{3}$.

It can be shown that we can paste for the whole block and obtain a weak realization which is a union of weak realizations of two copies of an orthomodular lattice given in Fig. 7.4. Hence, this suborthoposet has 200 elements (99 atoms) and a 58-element subset without any two-valued state.

It should be noted that in [17] there is an example of a 33-element set of lines without any two-valued state. Direction vectors of these lines arise by all permutations of coordinates from $(0,0,1),(0, \pm 1,1)(0, \pm 1, \sqrt{2}),( \pm 1, \pm 1, \sqrt{2})$. This set of lines orthogenerates a suborthoposet of $H_{3}$ with 116 elements (57 atoms). Direction vectors of remaining lines arise by all permutations of coordinates from $( \pm 1, \pm 3, \sqrt{2})$. This suborthoposet of $H_{3}$ has a 17 -element set of orthogenerators (e.g., lines with direction vectors $(0,0,1),(0,1,0)$ and all coordinate permutations from $(0,1, \sqrt{2})$, $(1, \pm 1, \sqrt{2}))$ and a three-element set of generators $[$ e.g., lines with direction vectors $(1,0,0),(1,1,0)$, $(\sqrt{2}, 1,1)$. The "almost" Greechie diagram (24 points that belong to exactly one edge are, for simplicity, omitted) of this example is given in Fig. 9 (one edge is denoted by a circle). The abovementioned three-element set of generators is marked by circles.

Corollary 7.5: There is a three-element set of lines in $\mathrm{H}_{3}$ such that no subortholattice of $\mathrm{H}_{3}$ containing it has a two-valued state. 


\begin{tabular}{|l|r|r|r|r|r|}
\hline "Large": & Full & Separating & \multicolumn{2}{|c|}{ Unital } & Nonempty \\
\hline Example (figure) & 4.2 & 7.2 & {$[24,27]$} & 8 & 9 \\
\hline \hline Elements of a suborthoposet & 28 & 56 & 76 & 104 & 116 \\
\hline Atoms of a suborthoposet & 13 & 27 & 37 & 51 & 57 \\
\hline Lines & 8 & 17 & 25 & 29 & 33 \\
\hline Orthogenerators & 6 & 10 & 11 & 16 & 17 \\
\hline Generators & 3 & 4 & 3 & 4 & 3 \\
\hline
\end{tabular}

TABLE I: Numbers of elements of constructed propositional structures in $H_{3}$ without a 'large' set of two-valued states.

It seems to be an open question whether every 3 -element set of mutually nonorthogonal lines in $H_{3}$ generates a subortholattice without any two-valued state. The least numbers in constructions are given in Table I.

Let us note that the examples in Proposition 7.1 and in Proposition 7.2 appeared in [12], the example in Fig. 7.4 appeared (not explicitly) in $[12,16]$ as a part of their construction. In [1] the author uses (not explicitly) the orthomodular lattice given in Fig. 7.3 and paste three copies to distinct atoms of a block obtaining thus an orthomodular lattice without any two-valued state (however, his estimation of lines does not seem to be correct).

In [13] the author uses weak realizability of an orthomodular lattice in Fig. 7.5 whenever we represent elements $a, b$ by lines in $H_{3}$ such that their angle is less than $25^{\circ}$. This leads to the construction of an orthomodular lattice with 392 elements (146 atoms) weakly realizable in $\mathrm{H}_{3}$ and (at most) 130-element set of lines without any two-valued state.

\section{DISCUSSION OF PHYSICAL RELEVANCE}

In this final section we shall give a brief review of the physical relevance of the above findings. The nonexistence of two-valued measures on certain finite propositional structures in threedimensional Hilbert spaces has first been explicitly demonstrated by Kochen and Specker [12]. It is strongly recommended that this original account be read. Their result has given rise to a number of interpretations, by Kochen and Specker and others. A detailed overview of the history of the subject can, for instance, be found in the reviews by Mermin [13] and Brown [3].

What does it physically mean that three nonorthogonal rays in three-dimensional Hilbert space are sufficient to generate a finite system of rays which have no two-valued state? To state the associated Kochen-Specker paradox explicitly, let us associate any one-dimensional subspace $\operatorname{Sp}(v)$ spanned by a nonzero vector $v$ with the proposition that the physical system is in a pure state associated with that subspace. That is,

$$
\mathrm{Sp}(1,0,0)=a, \quad \operatorname{Sp}(1,1,0)=b, \quad \operatorname{Sp}(\sqrt{2}, 1,1)=c,
$$

where $a, b$ and $c$ are propositions. If $a$ (similar for $b$ and $c$ ) is measured, then we associate the logical value "true" or "false" with the two-valued state function $s(a)=1$ and $s(a)=0$, respectively. $a, b, c$ generate the propositional structure derived by Peres [17, 18, pp. 186-190]. That is, if $v$ and $w$ are two vectors in three-dimensional Hilbert space corresponding to the propositions $p_{v}$ and $p_{w}$, respectively, then the vector product $v \times w$ corresponds to the proposition $\left(p_{v} \vee p_{w}\right)^{\prime}$. In particular,

$$
\begin{aligned}
\operatorname{Sp}(1,0,0) & =a \\
\operatorname{Sp}(1,1,0) & =b, \\
\operatorname{Sp}(\sqrt{2}, 1,1) & =c, \\
\operatorname{Sp}(0,0,1) & =(\operatorname{Sp}(1,0,0) \vee \operatorname{Sp}(1,1,0))^{\prime} \\
& =(a \vee b)^{\prime},
\end{aligned}
$$




$$
\begin{aligned}
& \operatorname{Sp}(0,1,-1)=(\operatorname{Sp}(1,0,0) \vee \operatorname{Sp}(\sqrt{2}, 1,1))^{\prime} \\
& =(a \vee c)^{\prime} \text {, } \\
& \operatorname{Sp}(0,1,0)=(\operatorname{Sp}(1,0,0) \vee \operatorname{Sp}(0,0,1))^{\prime} \\
& =\left(a \vee(a \vee b)^{\prime}\right)^{\prime} \text {, } \\
& \operatorname{Sp}(0,1,1)=(\operatorname{Sp}(1,0,0) \vee \operatorname{Sp}(0,1,-1))^{\prime} \\
& =\left(a \vee(a \vee c)^{\prime}\right)^{\prime} \text {, } \\
& \operatorname{Sp}(1,-1,0)=(\operatorname{Sp}(1,1,0) \vee \operatorname{Sp}(0,0,1))^{\prime} \\
& =\left(b \vee(a \vee b)^{\prime}\right)^{\prime} \text {, } \\
& \mathrm{Sp}(-1, \sqrt{2}, 0)=(\operatorname{Sp}(\sqrt{2}, 1,1) \vee \operatorname{Sp}(0,0,1))^{\prime} \\
& =\left(c \vee(a \vee b)^{\prime}\right)^{\prime} \text {, } \\
& \operatorname{Sp}(\sqrt{2},-1,-1)=(\operatorname{Sp}(\sqrt{2}, 1,1) \vee \operatorname{Sp}(0,1,-1))^{\prime} \\
& =\left(c \vee(a \vee c)^{\prime}\right)^{\prime} \text {, } \\
& \mathrm{Sp}(-1,0, \sqrt{2})=(\operatorname{Sp}(\sqrt{2}, 1,1) \vee \mathrm{Sp}(0,1,0))^{\prime} \\
& =\left(c \vee\left(a \vee(a \vee b)^{\prime}\right)^{\prime}\right)^{\prime} \text {, } \\
& \operatorname{Sp}(\sqrt{2}, 1,0)=(\operatorname{Sp}(0,0,1) \vee \operatorname{Sp}(-1, \sqrt{2}, 0))^{\prime} \\
& =\left((a \vee b)^{\prime} \vee\left(c \vee(a \vee b)^{\prime}\right)^{\prime}\right)^{\prime} \text {, } \\
& \operatorname{Sp}(1, \sqrt{2}, 0)=(\operatorname{Sp}(0,0,1) \vee \operatorname{Sp}(\sqrt{2},-1,-1))^{\prime} \\
& =\left((a \vee b)^{\prime} \vee\left(c \vee(a \vee c)^{\prime}\right)^{\prime}\right)^{\prime} \text {, } \\
& \operatorname{Sp}(1,0, \sqrt{2})=(\operatorname{Sp}(0,1,0) \vee \operatorname{Sp}(\sqrt{2},-1,-1))^{\prime} \\
& =\left(\left(a \vee(a \vee b)^{\prime}\right)^{\prime} \vee\left(c \vee(a \vee c)^{\prime}\right)^{\prime}\right)^{\prime} \text {, } \\
& \operatorname{Sp}(\sqrt{2}, 1,-1)=(\operatorname{Sp}(0,1,1) \vee \operatorname{Sp}(-1, \sqrt{2}, 0))^{\prime} \\
& =\left(\left(a \vee(a \vee c)^{\prime}\right)^{\prime} \vee\left(c \vee(a \vee b)^{\prime}\right)^{\prime}\right)^{\prime} \text {, } \\
& \operatorname{Sp}(\sqrt{2}, 0,1)=(\operatorname{Sp}(0,1,0) \vee \operatorname{Sp}(-1,0, \sqrt{2}))^{\prime} \\
& =\left(\left(a \vee(a \vee b)^{\prime}\right)^{\prime} \vee\left(c \vee\left(a \vee(a \vee b)^{\prime}\right)^{\prime}\right)^{\prime}\right)^{\prime} \text {, } \\
& \operatorname{Sp}(\sqrt{2},-1,0)=(\operatorname{Sp}(0,0,1) \vee \operatorname{Sp}(1, \sqrt{2}, 0))^{\prime} \\
& =\left((a \vee b)^{\prime} \vee\left((a \vee b)^{\prime} \vee\left(c \vee(a \vee c)^{\prime}\right)^{\prime}\right)^{\prime}\right)^{\prime} \text {, } \\
& \operatorname{Sp}(\sqrt{2},-1,1)=(\operatorname{Sp}(0,1,1) \vee \operatorname{Sp}(-1,0, \sqrt{2}))^{\prime} \\
& =\left(\left(a \vee(a \vee c)^{\prime}\right)^{\prime} \vee\left(c \vee\left(a \vee(a \vee b)^{\prime}\right)^{\prime}\right)^{\prime}\right)^{\prime} \text {, } \\
& \operatorname{Sp}(-1,1, \sqrt{2})=(\operatorname{Sp}(1,1,0) \vee \operatorname{Sp}(\sqrt{2}, 0,1))^{\prime} \\
& =\left(b \vee\left(\left(a \vee(a \vee b)^{\prime}\right)^{\prime} \vee\left(c \vee\left(a \vee(a \vee b)^{\prime}\right)^{\prime}\right)^{\prime}\right)^{\prime}\right)^{\prime} \text {, } \\
& \operatorname{Sp}(0, \sqrt{2},-1)=(\operatorname{Sp}(1,0,0) \vee \operatorname{Sp}(-1,1, \sqrt{2}))^{\prime} \\
& =\left(a \vee\left(b \vee\left(\left(a \vee(a \vee b)^{\prime}\right)^{\prime} \vee\left(c \vee\left(a \vee(a \vee b)^{\prime}\right)^{\prime}\right)^{\prime}\right)^{\prime}\right)^{\prime}\right)^{\prime} \text {, } \\
& \operatorname{Sp}(\sqrt{2}, 0,-1)=(\operatorname{Sp}(0,1,0) \vee \operatorname{Sp}(1,0, \sqrt{2}))^{\prime} \\
& =\left(\left(a \vee(a \vee b)^{\prime}\right)^{\prime} \vee\left(\left(a \vee(a \vee b)^{\prime}\right)^{\prime} \vee\left(c \vee(a \vee c)^{\prime}\right)^{\prime}\right)^{\prime}\right)^{\prime} \text {, } \\
& \operatorname{Sp}(1,-1, \sqrt{2})=(\operatorname{Sp}(1,1,0) \vee \operatorname{Sp}(-1,1, \sqrt{2}))^{\prime} \\
& =\left(b \vee\left(b \vee\left(\left(a \vee(a \vee b)^{\prime}\right)^{\prime} \vee\left(c \vee\left(a \vee(a \vee b)^{\prime}\right)^{\prime}\right)^{\prime}\right)^{\prime}\right)^{\prime}\right)^{\prime} \text {, } \\
& \operatorname{Sp}(0,1, \sqrt{2})=(\operatorname{Sp}(1,0,0) \vee \operatorname{Sp}(0, \sqrt{2},-1))^{\prime} \\
& =\left(a \vee\left(a \vee\left(b \vee\left(\left(a \vee(a \vee b)^{\prime}\right)^{\prime} \vee\left(c \vee\left(a \vee(a \vee b)^{\prime}\right)^{\prime}\right)^{\prime}\right)^{\prime}\right)^{\prime}\right)^{\prime}\right)^{\prime} \text {, } \\
& \operatorname{Sp}(0, \sqrt{2}, 1)=(\operatorname{Sp}(1,0,0) \vee \operatorname{Sp}(1,-1, \sqrt{2}))^{\prime} \\
& =\left(a \vee\left(b \vee\left(b \vee\left(\left(a \vee(a \vee b)^{\prime}\right)^{\prime} \vee\left(c \vee\left(a \vee(a \vee b)^{\prime}\right)^{\prime}\right)^{\prime}\right)^{\prime}\right)^{\prime}\right)^{\prime}\right)^{\prime} \text {, } \\
& \operatorname{Sp}(-1,-1, \sqrt{2})=(\operatorname{Sp}(1,-1,0) \vee \operatorname{Sp}(\sqrt{2}, 0,1))^{\prime} \\
& =\left(\left(b \vee(a \vee b)^{\prime}\right)^{\prime} \vee\left(\left(a \vee(a \vee b)^{\prime}\right)^{\prime} \vee\left(c \vee\left(a \vee(a \vee b)^{\prime}\right)^{\prime}\right)^{\prime}\right)^{\prime}\right)^{\prime} \text {, }
\end{aligned}
$$




$$
\begin{aligned}
& \operatorname{Sp}(0,-1, \sqrt{2})=(\operatorname{Sp}(1,0,0) \vee \operatorname{Sp}(0, \sqrt{2}, 1))^{\prime} \\
& =\left(a \vee\left(a \vee\left(b \vee\left(b \vee\left(\left(a \vee(a \vee b)^{\prime}\right)^{\prime} \vee\left(c \vee\left(a \vee(a \vee b)^{\prime}\right)^{\prime}\right)^{\prime}\right)^{\prime}\right)^{\prime}\right)^{\prime}\right)^{\prime}\right)^{\prime}, \\
& \operatorname{Sp}(1,1, \sqrt{2})=(\operatorname{Sp}(1,-1,0) \vee \operatorname{Sp}(0, \sqrt{2},-1))^{\prime} \\
& =\left(\left(b \vee(a \vee b)^{\prime}\right)^{\prime} \vee\left(a \vee\left(b \vee\left(\left(a \vee(a \vee b)^{\prime}\right)^{\prime} \vee\left(c \vee\left(a \vee(a \vee b)^{\prime}\right)^{\prime}\right)^{\prime}\right)^{\prime}\right)^{\prime}\right)^{\prime}\right)^{\prime} \text {, } \\
& \operatorname{Sp}(-1, \sqrt{2},-1)=(\operatorname{Sp}(\sqrt{2}, 1,0) \vee \operatorname{Sp}(0,1, \sqrt{2}))^{\prime} \\
& =\left(\left((a \vee b)^{\prime} \vee\left(c \vee(a \vee b)^{\prime}\right)^{\prime}\right)^{\prime} \vee\right. \\
& \left.\left(a \vee\left(a \vee\left(b \vee\left(\left(a \vee(a \vee b)^{\prime}\right)^{\prime} \vee\left(c \vee\left(a \vee(a \vee b)^{\prime}\right)^{\prime}\right)^{\prime}\right)^{\prime}\right)^{\prime}\right)^{\prime}\right)^{\prime}\right)^{\prime} \text {, } \\
& \operatorname{Sp}(-1, \sqrt{2}, 1)=(\operatorname{Sp}(\sqrt{2}, 1,0) \vee \operatorname{Sp}(0,-1, \sqrt{2}))^{\prime} \\
& =\left(\left((a \vee b)^{\prime} \vee\left(c \vee(a \vee b)^{\prime}\right)^{\prime}\right)^{\prime} \vee\right. \\
& \left.\left(a \vee\left(a \vee\left(b \vee\left(b \vee\left(\left(a \vee(a \vee b)^{\prime}\right)^{\prime} \vee\left(c \vee\left(a \vee(a \vee b)^{\prime}\right)^{\prime}\right)^{\prime}\right)^{\prime}\right)^{\prime}\right)^{\prime}\right)^{\prime}\right)^{\prime}\right)^{\prime} \text {, } \\
& \operatorname{Sp}(1, \sqrt{2},-1)=(\operatorname{Sp}(\sqrt{2},-1,0) \vee \operatorname{Sp}(0,1, \sqrt{2}))^{\prime} \\
& =\left(\left((a \vee b)^{\prime} \vee\left((a \vee b)^{\prime} \vee\left(c \vee(a \vee c)^{\prime}\right)^{\prime}\right)^{\prime}\right)^{\prime} \vee\right. \\
& \left.\left(a \vee\left(a \vee\left(b \vee\left(\left(a \vee(a \vee b)^{\prime}\right)^{\prime} \vee\left(c \vee\left(a \vee(a \vee b)^{\prime}\right)^{\prime}\right)^{\prime}\right)^{\prime}\right)^{\prime}\right)^{\prime}\right)^{\prime}\right)^{\prime} \text {, } \\
& \operatorname{Sp}(-1,0,1)=(\operatorname{Sp}(0,1,0) \vee \mathrm{Sp}(-1, \sqrt{2},-1))^{\prime} \\
& =\left(( a \vee ( a \vee b ) ^ { \prime } ) ^ { \prime } \vee \left(\left((a \vee b)^{\prime} \vee\left(c \vee(a \vee b)^{\prime}\right)^{\prime}\right)^{\prime} \vee\right.\right. \\
& \left.\left.\left(a \vee\left(a \vee\left(b \vee\left(\left(a \vee(a \vee b)^{\prime}\right)^{\prime} \vee\left(c \vee\left(a \vee(a \vee b)^{\prime}\right)^{\prime}\right)^{\prime}\right)^{\prime}\right)^{\prime}\right)^{\prime}\right)^{\prime}\right)^{\prime}\right)^{\prime} \text {, } \\
& \operatorname{Sp}(1, \sqrt{2}, 1)=(\operatorname{Sp}(\sqrt{2},-1,0) \vee \operatorname{Sp}(0,-1, \sqrt{2}))^{\prime} \\
& =\left(\left((a \vee b)^{\prime} \vee\left((a \vee b)^{\prime} \vee\left(c \vee(a \vee c)^{\prime}\right)^{\prime}\right)^{\prime}\right)^{\prime} \vee\right. \\
& \left.\left(a \vee\left(a \vee\left(b \vee\left(b \vee\left(\left(a \vee(a \vee b)^{\prime}\right)^{\prime} \vee\left(c \vee\left(a \vee(a \vee b)^{\prime}\right)^{\prime}\right)^{\prime}\right)^{\prime}\right)^{\prime}\right)^{\prime}\right)^{\prime}\right)^{\prime}\right)^{\prime} \text {, } \\
& \operatorname{Sp}(1,0,1)=(\operatorname{Sp}(0,1,0) \vee \operatorname{Sp}(-1, \sqrt{2}, 1))^{\prime} \\
& =\left(( a \vee ( a \vee b ) ^ { \prime } ) ^ { \prime } \vee \left(\left((a \vee b)^{\prime} \vee\left(c \vee(a \vee b)^{\prime}\right)^{\prime}\right)^{\prime} \vee\right.\right. \\
& \left.\left.\left(a \vee\left(a \vee\left(b \vee\left(b \vee\left(\left(a \vee(a \vee b)^{\prime}\right)^{\prime} \vee\left(c \vee\left(a \vee(a \vee b)^{\prime}\right)^{\prime}\right)^{\prime}\right)^{\prime}\right)^{\prime}\right)^{\prime}\right)^{\prime}\right)^{\prime}\right)^{\prime}\right)^{\prime}
\end{aligned}
$$

Suppose, for the sake of contradiction, that each one of the above 33 propositions corresponds to an "element of physical reality" [7]. That is, suppose that its value is either "true" (exclusive) or "false," irrespective of whether it has been actually measured or just counterfactually inferred. Let us further assume with Peres [17, 18, pp. 186-190] that-provided these "elements of reality" exist- $\operatorname{Sp}(0,0,1)=\operatorname{Sp}(1,0,1)=\operatorname{Sp}(0,1,1)=\operatorname{Sp}(1,-1, \sqrt{2})=\operatorname{Sp}(1,0, \sqrt{2})=\operatorname{Sp}(\sqrt{2}, 1,1)=$ $\operatorname{Sp}(\sqrt{2}, 0,1)=\operatorname{Sp}(1,1, \sqrt{2})=\operatorname{Sp}(0,1, \sqrt{2})=\operatorname{Sp}(1, \sqrt{2}, 1)=$ "true." One can follow Peres' arguments to show that - provided these "elements of reality" exist - all other rays belong to triads which are orthogonal to the above rays. Therefore, these latter rays must correspond to propositions whose value is "false." In particular, $\operatorname{Sp}(1,0,0)=\operatorname{Sp}(0, \sqrt{2}, 1)=\operatorname{Sp}(0,-1, \sqrt{2})=$ "false," associate with $s(\operatorname{Sp}(1,0,0))=s(\operatorname{Sp}(0, \sqrt{2}, 1))=s(\operatorname{Sp}(0,-1, \sqrt{2}))=0$. Thus, $s(\operatorname{Sp}(1,0,0))+s(\operatorname{Sp}(0, \sqrt{2}, 1))+$ $s(\operatorname{Sp}(0,-1, \sqrt{2}))=0+0+0=0$. But $\operatorname{Sp}(1,0,0), \operatorname{Sp}(0, \sqrt{2}, 1)$ and $\operatorname{Sp}(0,-1, \sqrt{2})$ are mutually orthogonal. This is in contradiction to the assumption that for any orthogonal triad spanning the entire Hilbert space, the sum of the measures should be one [cf. Definition 3.1.(4)]. Notice that in order to arrive at this Kochen-Specker paradox, we had to explicitly assume the existence of the "elements of reality," irrespective of whether they have (or could have) actually been measured or not.

What physical use can be a paradox? How can one measure a contradiction? Indeed, what can actually be measured is merely one triplet of propositions corresponding to some of the triads of mutually orthogonal rays. Such a measurement can be performed with the operator discussed by Peres, or with an arrangement of beam splitters discussed by Reck et al. [20].

For instance, after $c$ is found to be "true" [corresponding to $s(c)=1$ ], then measurement of the original values of $a$ or $b$ is no longer possible. However, suppose one would be willing to believe in the existence of "elements of reality" [7,15], which could merely be counterfactually inferred. Then one could for instance - at least in principle - "measure" all 16 orthogonal triads by the production 
of a state with 16 entangled subsystems. On each one of the 16 different entangled subsystems one could measure one of the 16 different orthogonal triads. This is similar to a proposal by Greenberger, Horne and Zeilinger [6], which use three particles and eight-dimensional Hilbert space. Indeed, only in such a way - namely by (counterfactually) inferring noncomeasurable propositions - one would encounter a complete Kochen-Specker contradiction.

As has been already proven in Kochen and Specker's original work [12, pp. 82-85, Theorem 4], the notion of tautology is connected to a classical (Boolean) imbedding of a partial Boolean algebra. Indeed, there exist propositions which are tautologies in the classical (Boolean) algebra but which are not tautologies in the partial Boolean algebra if and only if the partial Boolean algebra does not have a unital set of two-valued states and thus cannot be imbedded into a classical (Boolean) algebra.

This is true for all partial Boolean algebras, in particular for orthomodular posets. Notice that the above result does not imply that every propositional structure giving rise to a (classical) Boolean tautology which is no quantum tautology also has no two-valued measure (cf. below).

Until now, the lowest number of rays necessary to produce a classical tautology which is not always true quantum mechanically is due to Schütte $[24,27]$. The 11 rays used by Schütte can also be generated by the three vectors $(1,0,0),(1,1,0)$ and $(\sqrt{2}, 1,1)$ (corresponding to $a, b$ and $c$ ) used before. Indeed, $d=\operatorname{Sp}(0,1,-1)=(\operatorname{Sp}(1,1,0) \vee \operatorname{Sp}(\sqrt{2}, 1,1))^{\prime}=(a \vee c)^{\prime}$ and

$$
\begin{aligned}
a_{1} & =\operatorname{Sp}(1,0,0)=a \\
a_{2} & =\operatorname{Sp}(0,1,0)=(\operatorname{Sp}(1,0,0) \vee \operatorname{Sp}(0,0,1))^{\prime} \\
& =\left(a \vee(a \vee b)^{\prime}\right)^{\prime} \\
b_{1} & =\operatorname{Sp}(0,1,1)=(\operatorname{Sp}(1,0,0) \vee \operatorname{Sp}(0,1,-1))^{\prime} \\
& =(a \vee d)^{\prime} \\
b_{2} & =\operatorname{Sp}(1,0,1)=(\operatorname{Sp}(0,1,0) \vee \operatorname{Sp}(-1,1,1))^{\prime} \\
& =\left(\left(a \vee(a \vee b)^{\prime}\right)^{\prime} \vee(b \vee d)^{\prime}\right)^{\prime} \\
b_{3} & =\operatorname{Sp}(1,1,0)=b \\
c_{1} & =\operatorname{Sp}(1,0,2)=(\operatorname{Sp}(0,1,0) \vee \operatorname{Sp}(2,1,-1))^{\prime} \\
& =\left(\left(a \vee(a \vee b)^{\prime}\right)^{\prime} \vee\left((a \vee d)^{\prime} \vee\left(b \vee(a \vee d)^{\prime}\right)^{\prime}\right)^{\prime}\right)^{\prime}, \\
c_{2} & =\operatorname{Sp}(2,0,1)=(\operatorname{Sp}(0,1,0) \vee \operatorname{Sp}(-1,0,2))^{\prime} \\
& =\left(\left(a \vee(a \vee b)^{\prime}\right)^{\prime} \vee\left(\left(a \vee(a \vee b)^{\prime}\right)^{\prime} \vee\left((a \vee d)^{\prime} \vee\left((a \vee d)^{\prime} \vee\left(b \vee(a \vee b)^{\prime}\right)^{\prime}\right)^{\prime}\right)^{\prime}\right)^{\prime}\right)^{\prime}, \\
d_{1} & =\operatorname{Sp}(-1,1,1)=(\operatorname{Sp}(1,1,0) \vee \operatorname{Sp}(0,1,-1))^{\prime} \\
& =(b \vee d)^{\prime}, \\
d_{2} & =\operatorname{Sp}(1,-1,1)=(\operatorname{Sp}(1,1,0) \vee \operatorname{Sp}(0,1,1))^{\prime} \\
& =\left(b \vee(a \vee d)^{\prime}\right)^{\prime}, \\
d_{3} & =\operatorname{Sp}(1,1,-1)=(\operatorname{Sp}(0,1,1) \vee \operatorname{Sp}(1,-1,0))^{\prime} \\
& =\left((a \vee d)^{\prime} \vee\left(b \vee(a \vee b)^{\prime}\right)^{\prime}\right)^{\prime}, \\
d_{4} & =\operatorname{Sp}(1,1,1)=(\operatorname{Sp}(0,1,-1) \vee \operatorname{Sp}(1,-1,0))^{\prime} \\
& =\left(d \vee\left(b \vee(a \vee b)^{\prime}\right)^{\prime}\right)^{\prime},
\end{aligned}
$$

where

$$
\begin{aligned}
\operatorname{Sp}(2,1,-1)= & (\mathrm{Sp}(0,1,1) \vee \operatorname{Sp}(1,-1,1))^{\prime}= \\
& \left((a \vee d)^{\prime} \vee\left(b \vee(a \vee d)^{\prime}\right)^{\prime}\right)^{\prime}, \\
\mathrm{Sp}(-1,0,2)= & (\mathrm{Sp}(0,1,0) \vee \mathrm{Sp}(-2,1,-1))^{\prime}= \\
& \left(\left(a \vee(a \vee b)^{\prime}\right)^{\prime} \vee\left((a \vee d)^{\prime} \vee\left((a \vee d)^{\prime} \vee\left(b \vee(a \vee b)^{\prime}\right)^{\prime}\right)^{\prime}\right)^{\prime}\right)^{\prime}, \\
\mathrm{Sp}(2,-1,1)= & (\mathrm{Sp}(0,1,1) \vee \mathrm{Sp}(1,1,-1))^{\prime}= \\
& \left((a \vee d)^{\prime} \vee\left((a \vee d)^{\prime} \vee\left(b \vee(a \vee b)^{\prime}\right)^{\prime}\right)^{\prime}\right)^{\prime} .
\end{aligned}
$$


As we have mentioned above, there is not a unital set of two-valued states on a suborthoposet orthogenerated by these rays (e.g., there is no two-valued state $s$ with $s(\operatorname{Sp}(1,0,0))=1)$. On the other hand, a two-valued state can be defined by $s(\operatorname{Sp}(0,1,0))=s(\operatorname{Sp}(0,1,1))=s(\operatorname{Sp}(1,1,0))=$ $s(\operatorname{Sp}(1,1,1))=s(\operatorname{Sp}(1,1,2))=s(\operatorname{Sp}(1,2,1))=s(\operatorname{Sp}(2,1,1))=s(\operatorname{Sp}(1,2,-1))=s(\operatorname{Sp}(-1,2,1))=$ $s(\mathrm{Sp}(1,5,2))=s(\operatorname{Sp}(2,5,1))=s(\mathrm{Sp}(-1,5,2))=s(\operatorname{Sp}(2,5,-1))=s(\operatorname{Sp}(1,5,-2))=$ $s(\operatorname{Sp}(-2,5,1))=1$ and $s(\operatorname{Sp}(1,0,0))=s(\operatorname{Sp}(0,0,1))=s(\operatorname{Sp}(1,0,1))=s(\operatorname{Sp}(0,1,-1))=$ $s(\operatorname{Sp}(1,0,-1))=s(\operatorname{Sp}(1,-1,0))=s(\operatorname{Sp}(1,1,-1))=s(\operatorname{Sp}(1,-1,1))=s(\operatorname{Sp}(-1,1,1))=$ $s(\operatorname{Sp}(-1,-1,2))=s(\operatorname{Sp}(-1,2,-1))=s(\operatorname{Sp}(2,-1,-1))=s(\operatorname{Sp}(1,-1,2))=s(\operatorname{Sp}(-1,1,2))=$ $s(\operatorname{Sp}(2,1,-1))=s(\operatorname{Sp}(2,-1,1))=s(\operatorname{Sp}(1,0,2))=s(\operatorname{Sp}(2,0,1))=s(\operatorname{Sp}(-1,0,2))=$ $s(\operatorname{Sp}(2,0,-1))=s(\operatorname{Sp}(1,-5,2))=s(\operatorname{Sp}(2,-5,1))=0$.

Consider now the following propositions (notice that any binary operation is either performed by orthogonal rays or by a ray and an orthocomplement of another ray such that these rays are orthogonal):

$$
\begin{aligned}
f_{1} & =d_{1} \rightarrow b_{2}^{\prime}=\left(d_{1} \wedge b_{2}\right)^{\prime} \\
f_{2} & =d_{1} \rightarrow b_{3}^{\prime}=\left(d_{1} \wedge b_{3}\right)^{\prime} \\
f_{3} & =d_{2} \rightarrow a_{2} \vee b_{2}=\left(d_{2} \wedge\left(a_{2} \vee b_{2}\right)^{\prime}\right)^{\prime} \\
f_{4} & =d_{2} \rightarrow b_{3}^{\prime}=\left(d_{2} \wedge b_{3}\right)^{\prime} \\
f_{5} & =d_{3} \rightarrow b_{2}^{\prime}=\left(d_{3} \wedge b_{2}\right)^{\prime} \\
f_{6} & =d_{3} \rightarrow\left(a_{1} \vee a_{2} \rightarrow b_{3}\right)=\left(d_{3} \wedge\left(\left(a_{1} \vee a_{2}\right)^{\prime} \vee b_{3}\right)^{\prime}\right)^{\prime} \\
f_{7} & =d_{4} \rightarrow a_{2} \vee b_{2}=\left(d_{4} \wedge\left(a_{2} \vee b_{2}\right)^{\prime}\right)^{\prime} \\
f_{8} & =d_{4} \rightarrow\left(a_{1} \vee a_{2} \rightarrow b_{3}\right)=\left(d_{4} \wedge\left(\left(a_{1} \vee a_{2}\right)^{\prime} \vee b_{3}\right)^{\prime}\right)^{\prime} \\
f_{9} & =\left(a_{2} \vee c_{1}\right) \vee\left(b_{3} \vee d_{1}\right)=\left(\left(a_{2} \vee c_{1}\right)^{\prime} \wedge\left(b_{3} \vee d_{1}\right)^{\prime}\right)^{\prime} \\
f_{10} & =\left(a_{2} \vee c_{2}\right) \vee\left(a_{1} \vee b_{1} \rightarrow d_{1}\right)=\left(\left(a_{2} \vee c_{2}\right)^{\prime} \wedge\left(\left(a_{1} \vee b_{1}\right)^{\prime} \vee d_{1}\right)^{\prime}\right)^{\prime} \\
f_{11} & =c_{1} \rightarrow b_{1} \vee d_{2}=\left(c_{1} \wedge\left(b_{1} \vee d_{2}\right)^{\prime}\right)^{\prime} \\
f_{12} & =c_{2} \rightarrow b_{3} \vee d_{2}=\left(c_{2} \wedge\left(b_{3} \vee d_{2}\right)^{\prime}\right)^{\prime} \\
f_{13} & =\left(a_{2} \vee c_{1}\right) \vee\left[\left(a_{1} \vee a_{2} \rightarrow b_{3}\right) \rightarrow d_{3}\right]=\left(\left(a_{2} \vee c_{1}\right)^{\prime} \wedge\left(\left(\left(a_{1} \vee a_{2}\right)^{\prime} \vee b_{3}\right)^{\prime} \vee d_{3}\right)^{\prime}\right)^{\prime} \\
f_{14} & =\left(a_{2} \vee c_{2}\right) \vee\left(b_{1} \vee d_{3}\right)=\left(\left(a_{2} \vee c_{2}\right)^{\prime} \wedge\left(b_{1} \vee d_{3}\right)^{\prime}\right)^{\prime} \\
f_{15} & =c_{2} \rightarrow\left[\left(a_{1} \vee a_{2} \rightarrow b_{3}\right) \rightarrow d_{4}\right]=\left(c_{2} \wedge\left(\left(\left(a_{1} \vee a_{2}\right)^{\prime} \vee b_{3}\right)^{\prime} \vee d_{4}\right)^{\prime}\right)^{\prime} \\
f_{16} & =c_{1} \rightarrow\left(a_{1} \vee b_{1} \rightarrow d_{4}\right)=\left(c_{1} \wedge\left(\left(a_{1} \vee b_{1}\right)^{\prime} \vee d_{4}\right)^{\prime}\right)^{\prime} \\
f_{17} & =\left(a_{1} \rightarrow a_{2}\right) \vee b_{1}=\left(a_{1}^{\prime} \vee a_{2}\right) \vee b_{1} .
\end{aligned}
$$

The "implication" relation has been expressed as $x \rightarrow y \equiv x^{\prime} \vee y \equiv\left(x \wedge y^{\prime}\right)^{\prime}$.

As can be straightforwardly checked, the proposition formed by

$$
F: f_{1} \wedge f_{2} \wedge \cdots \wedge f_{16} \rightarrow f_{17}
$$

is a classical tautology. Nevertheless, $F$ is not valid in three-dimensional (real) Hilbert space $\mathbf{R}^{3}$, since $f_{1}, f_{2}, \ldots, f_{16}=\mathbf{R}^{3}$, whereas $f_{17}=(\operatorname{Sp}(1,0,0))^{\prime}=\operatorname{Sp}(0,1,0) \vee \operatorname{Sp}(0,0,1) \neq \mathbf{R}^{3}$.

The three vectors $(1,0,0),(1,1,0)$ and $(\sqrt{2}, 1,1)$ generating the Schütte rays are not mutually orthogonal. Therefore, the corresponding propositions $a, b$ and $c$ are not comeasurable. In the sense of partial algebras, they cannot be combined by logical operations "or" $(\vee)$, "and" $(\wedge)$, "not" (') to form new expressions. Thus, it would be incorrect to state that there exists a classical tautology in the three variables $a, b$ and $c$ which is no quantum tautology. Indeed, Coray proved [4] that all classical tautologies in three variables are tautologies in all partial algebras, in particular in the one associated with the logic of quantum observables.

However, also Schütte's example is counterfactual in nature. Although every operation or relation is solely defined on comeasurable propositions, the entire formula $F$ contains 11 noncomeasurable variables (nonorthogonal rays). In order to be able to evaluate this formula, one would have to know the truth value of all these 11 variables in parallel. Since they are not comeasurable, this is possible only by counterfactual inference; in very much the same way as discussed before in the case 
of the original Kochen-Specker paradox. Indeed, Corey's result shows that any classical (Boolean) tautology that is no quantum tautology will have to rely on at least four variables which cannot be mutually orthogonal (in $H_{3}$ ), and therefore must be based upon counterfactual inference.

Finally, let us shortly mention the relevance of these findings to the partition logic of automata. Corollary 4.3 states that every finite subortholattice of $H_{3}$ has a full (and thus separating) set of two-valued states. Thus, any finite subortholattice of $H_{3}$ can be expressed as an automaton logic. The subortholattices of $H_{3}$ which have no two-valued state are infinite.

\section{ACKNOWLEDGEMENT}

This research has been partially supported by the Austrian-Czech program AKTION.

\section{REFERENCES}

[1] V. Alda: On 0-1 measure for projectors. Apl. Mat. 25 (1980), 373-374.

[2] G. Birkhof and J. von Neumann: The logic of quantum mechanics. Ann. Math. 37 (1936), 823-834.

[3] H. R. Brown: Bell's other theorem and its connection with nonlocality, part I, in Bell's Theorem and the Foundations of Modern Physics, edited by A. Van der Merwe, F. Selleri and G. Tarozzi (World Scientific, Singapore, 1992), 104-116.

[4] G. Coray: Validité dans les algébres de Boole partielles. Commun. Math. Helv. 45 (1970), 49-82.

[5] A. Gleason: Measures on a closed subspaces of a Hilbert space. J. Math. Mech. 6 (1957), 883-894.

[6] D. M. Greenberger, M. Horne and A. Zeilinger: in Bell's Theorem, Quantum Theory, and Conceptions of the Universe, ed. by M. Kafatos (Kluwer, Dordrecht, 1989); D. M. Greenberger, M. A. Horne, A. Shimony and A. Zeilinger, Am. J. Phys. 58 (1990), 1131.

[7] A. Einstein, B. Podolsky and N. Rosen: Can quantum-mechanical description of physical reality be considered complete?. Phys. Rev. 47 (1935), 777; reprinted in J. A. Wheeler and W. H. Zurek, eds., Quantum Theory and Measurement (Princeton University Press, Princeton, 1983), $145-151$.

[8] G. Kalmbach: Orthomodular Lattices (Academic Press, New York, 1983).

[9] G. Kalmbach: Measures and Hilbert Lattices. World Scientific, Singapore, 1986.

[10] S. Kochen and E. P. Specker: Logical Structures arising in quantum theory, in Symposium on the Theory of Models, Proceedings of the 1963 International Symposium at Berkeley (NorthHolland, Amsterdam, 1965), 177-189.

[11] S. Kochen and E. P. Specker: The calculus of partial propositional functions, in Proceedings of the 1964 International Congress for Logic, Methodology and Philosophy of Science, Jerusalem (North-Holland, Amsterdam, 1965), 45-57.

[12] S. Kochen and E. P. Specker: The problem of hidden variables in quantum mechanics. J. Math. Mech. 17 (1967), 59-87, reprinted in E. Specker, Selecta (Birkhäuser Verlag, Basel, 1990)

[13] N. D. Mermin: Hidden variables and the two theorems of John Bell. Rev. Mod. Phys. 65 (1993), 803-815. 
[14] M. Navara, V. Rogalewicz: The pasting constructions for orthomodular posets. Math. Nachr. 154 (1991), 157-168.

[15] A. Peres: Unperformed experiments have no results. Am. J. Phys. 46 (1978), 745.

[16] A. Peres, A. Ron: Cryptodeterminism and quantum theory. In Microphysical Reality and Quantum Formalism, edited by A. van der Merwe (Kluwer Academic Publishers, Dordrecht, 1988), $115-123$.

[17] A. Peres: Two simple proofs of the Kochen-Specker theorem. J. Phys. A: Math. Gen. 24 (1991), L175-L178.

[18] A. Peres: Quantum Theory: Concepts and Methods (Kluwer Academic Publishers, Dordrecht, 1993).

[19] P. Pták and S. Pulmannová: Orthomodular Structures as Quantum Logics. (Kluwer Academic Publishers, Dordrecht, 1991).

[20] M. Reck, A. Zeilinger, H. J. Bernstein and P. Bertani: Experimental realization of any discrete unitary operator. Phys. Rev. Lett. 73 (1894), 58; see also F. D. Murnaghan, The Unitary and Rotation Groups (Spartan Books, Washington, 1962).

[21] M. Schaller and K. Svozil: Partition logics of automata. Nuovo Cimento B 109 (1994), 167176.

[22] M. Schaller and K. Svozil: Automaton partition logic versus quantum logic. Int. J. Theor. Phys. 34 (1995), 1741-1749.

[23] M. Schaller and K. Svozil: Automaton logic. Int. J. Theor. Phys. 35 (1996), 911-940.

[24] K. Schütte: letter to Professor E. P. Specker, dated April 22nd, 1965; published in E. Clavadetscher-Seeberger: Eine partielle Prädikatenlogik. Ph.D. thesis, ETH-Zürich, Zürich, 1983.

[25] E. P. Specker: Die Logik nicht gleichzeitig entscheidbarer Aussagen. Dialectica 14 (1960), 175182.

[26] K. Svozil: Randomness 86 Undecidability in Physics (World Scientific, Singapore, 1993).

[27] K. Svozil: A constructivist manifesto for the physical sciences, in The Foundational Debate, Complexity and Constructivity in Mathematics and Physics, edited by W. D. Schimanovich, E. Köhler and F. Stadler (Kluwer, Dordrecht, 1995), 65-88. 